\title{
Dispersive effective models for waves in heterogeneous media
}

Agnes Lamacz

Preprint 2010-05

März 2010

Fakultät für Mathematik

Technische Universität Dortmund

Vogelpothsweg 87

44227 Dortmund 



\title{
Dispersive effective models for waves in heterogeneous media
}

\author{
Agnes Lamacz ${ }^{1}$
}

March 22, 2010

\begin{abstract}
We study the long time behavior of waves in a strongly heterogeneous medium, starting from the one-dimensional scalar wave equation with variable coefficients. We assume that the coefficients are periodic with period $\varepsilon$ and $\varepsilon>0$ is a small length parameter. Our main result is the rigorous derivation of two different dispersive models. The first is a fourth-order equation with constant coefficients including powers of $\varepsilon$. In the second model, the $\varepsilon$-dependence is completely avoided by considering a third-order linearized Korteweg-de-Vries equation. Our result is that both simplified models describe the long time behavior well. An essential tool in our analysis is an adaption operator which modifies smooth functions according to the periodic structure of the medium.
\end{abstract}

Keywords: Homogenization, wave equation, dispersive model, long time behavior

\section{Introduction}

In recent years, many approaches have been developed for the homogenization of waves in periodic media. Powerful methods such as two-scale convergence (Allaire, [1]), the method of oscillating test-functions (Tartar) or compensated compactness, see [11], have been used to prove rigorous convergence results. By now, it is well known that, analogous to elliptic problems, the homogenization limit $(\varepsilon \rightarrow 0)$ of

$$
\partial_{\tau}^{2} \bar{u}_{\varepsilon}(x, \tau)=\partial_{x}\left(a\left(\frac{x}{\varepsilon}\right) \partial_{x} \bar{u}_{\varepsilon}(x, \tau)\right)
$$

is a linear wave equation with a constant coefficient $a^{*}$, called the effective coefficient. In other words, the problem fits the following picture: On bounded domains and short time intervals $\tau \in(0, T)$, the original solution $\bar{u}_{\varepsilon}$ coincides up to corrections of order $O(\varepsilon)$ with a limit function $\bar{u}$, which solves the effective wave equation $\partial_{\tau}^{2} \bar{u}-a^{*} \partial_{x}^{2} \bar{u}=0$.

\footnotetext{
${ }^{1}$ Technische Universität Dortmund, Fakultät für Mathematik, Vogelpothsweg 87, D-44227 Dortmund, Germany agnes.lamacz@tu-dortmund.de
} 
Nevertheless, the limit $\bar{u}_{\varepsilon} \rightarrow \bar{u}$ is a weak convergence and, in particular, the energy of the $\varepsilon$-solutions need not converge to the energy of $\bar{u}$, see [4].

In the present work we are interested in very long time scales, i.e. observation times of order $O\left(1 / \varepsilon^{2}\right)$. Numerical results and formal asymptotic expansions, see [6], suggest that the shape of the propagating wave is considerably modified at long sight. Our aim is to catch this effect with a uniformly valid dispersive model. The difficulty is, taking into account the long time scale $t:=\varepsilon^{2} \tau$, that we are now dealing with a true three-scale problem whose homogenization limit is not given by the solution to the effective wave equation. The interest in this question is not new, see [2] for dispersive limits in the case of large potentials and [3] in the case of high frequency initial data. Likewise, works such as $[7,8,9,12]$ deal with the long time behavior of waves and give formal calculations. Nevertheless, it seems that a rigorous mathematical statement (or even an effective equation) is still missing. With this paper, we would like to fill this gap by proposing two different dispersive models and proving that they both are well-posed and approximate the original problem for long observation times. While the first model (weakly dispersive equation) still depends on powers of $\varepsilon$, the second model (linearized Korteweg-de-Vries equation) is $\varepsilon$-independent. The general concept of our homogenization proofs can be described with the following three steps. 1) We state and solve the homogenized system. 2) We modify the solution of the homogenized system to construct an approximate solution to the original system. 3) We show by a testing procedure that the result of this construction is close to the original solution. This principle is flexible and can be applied in complex applications, e.g. to another three-scale problem in [13] or to problems with hysteresis in $[14,15,16]$.

Let us start with a detailed description of the original problem. We assume that the coefficient $a(y)$ is a real, smooth and periodic positive function with period $Y=(0,1)$. To be more precise, we assume that there exist $\alpha, \beta>0$ such that $a(y) \in C^{\infty}(\mathbb{R})$ with $0<\alpha \leq a(y) \leq \beta$ and $a(y+1)=a(y) \forall y$. Moreover, we are dealing with smooth initial data with compact support being perturbated at order $O(\varepsilon)$ by high frequency terms. To sum up, we consider the following problem on the long-time interval $\left(0, T / \varepsilon^{2}\right)$.

Definition 1.1 (Homogenization problem). Let $T, R>0$. Denote by $\bar{u}_{\varepsilon}(x, t)$ the unique solution to the wave equation

$$
\begin{aligned}
\partial_{\tau}^{2} \bar{u}_{\varepsilon}(x, \tau) & =\partial_{x}\left(a\left(\frac{x}{\varepsilon}\right) \partial_{x} \bar{u}_{\varepsilon}(x, \tau)\right), \\
\bar{u}_{\varepsilon}(x, 0) & =c_{0}(x)+\varepsilon L_{1}\left(\frac{x}{\varepsilon}\right) \partial_{x} c_{0}(x)+\varepsilon^{2} L_{2}\left(\frac{x}{\varepsilon}\right) \partial_{x}^{2} c_{0}(x), \\
\partial_{\tau} \bar{u}_{\varepsilon}(x, 0) & =d_{0}(x)+\varepsilon L_{1}\left(\frac{x}{\varepsilon}\right) \partial_{x} d_{0}(x)
\end{aligned}
$$

for $(x, \tau) \in \mathbb{R} \times\left(0, T / \varepsilon^{2}\right)$. We assume that the initial data are smooth, $c_{0}, d_{0} \in$ $C_{c}^{\infty}((-R, R))$. The functions $L_{1}(y), L_{2}(y) \in C^{2}(\mathbb{R})$ are $Y$-periodic and solve auxiliary cell problems. They are defined in Definition 5.1.

Here, $C_{c}^{\infty}((-R, R))$ as usually denotes the set of all infinitely differentiable functions with compact support. Let us remark that the existence of a unique weak solu- 
tion $\bar{u}_{\varepsilon} \in L^{\infty}\left(0, T / \varepsilon^{2} ; H^{2}(\mathbb{R})\right)$, with $\partial_{\tau} \bar{u}_{\varepsilon} \in L^{\infty}\left(0, T / \varepsilon^{2} ; H^{1}(\mathbb{R})\right)$, to the homogenization problem is a classical result.

\section{Rescaled problem and main results}

In this section we introduce three different long time problems and state our main results. We start with a time-scaled version of the homogenization problem. Considering the long time variable $t:=\varepsilon^{2} \tau$ and setting $u_{\varepsilon}(x, t):=\bar{u}_{\varepsilon}\left(x, t / \varepsilon^{2}\right)$, one arrives at the following definition.

Definition 2.1 (Time-scaled homogenization problem). Let $(x, t) \in \mathbb{R} \times(0, T)$. Denote by $u_{\varepsilon}(x, t)$ the unique solution to

$$
\begin{aligned}
\varepsilon^{4} \partial_{t}^{2} u_{\varepsilon}(x, t) & =\partial_{x}\left(a\left(\frac{x}{\varepsilon}\right) \partial_{x} u_{\varepsilon}(x, t)\right), \\
u_{\varepsilon}(x, 0) & =c_{0}(x)+\varepsilon L_{1}\left(\frac{x}{\varepsilon}\right) \partial_{x} c_{0}(x)+\varepsilon^{2} L_{2}\left(\frac{x}{\varepsilon}\right) \partial_{x}^{2} c_{0}(x), \\
\partial_{t} u_{\varepsilon}(x, 0) & =\frac{1}{\varepsilon^{2}}\left(d_{0}(x)+\varepsilon L_{1}\left(\frac{x}{\varepsilon}\right) \partial_{x} d_{0}(x)\right) .
\end{aligned}
$$

Let us discuss here what we expect in view of the classical homogenization results. The effective coefficient $a^{*}$ is, in the one-dimensional case, given by the harmonic mean

$$
a^{*}=\left(\int_{Y} \frac{1}{a(y)} d y\right)^{-1} .
$$

The associated homogenized wave speed is $c^{*}:=\sqrt{a^{*}}$. Homogenization hence suggests that waves $\bar{u}_{\varepsilon}$ move with speed $c^{*}$. Accordingly, in the time-scaled version of Definition 2.1 , we expect that waves $u_{\varepsilon}$ propagate with an asymptotic speed $c^{*} / \varepsilon^{2}$.

In the next step, we introduce a fourth-order weakly dispersive solution. A proof of existence and uniqueness as well as energy estimates can be found in Section 4 .

Definition 2.2 (Weakly dispersive problem). Denote by $v_{\varepsilon}(x, t)$ the unique solution to the following problem

$$
\begin{aligned}
\varepsilon^{4} \partial_{t}^{2} v_{\varepsilon}(x, t) & -a^{*} \partial_{x}^{2} v_{\varepsilon}(x, t)-\varepsilon^{6} \frac{a_{2}^{*}}{a^{*}} \partial_{t}^{2} \partial_{x}^{2} v_{\varepsilon}(x, t)=0 \\
v_{\varepsilon}(x, 0) & =c_{0}(x) \\
\partial_{t} v_{\varepsilon}(x, 0) & =\frac{1}{\varepsilon^{2}} d_{0}(x)
\end{aligned}
$$

for $(x, t) \in \mathbb{R} \times(0, T)$ and $a_{2}^{*}>0$ introduced in Definition 5.1.

Finally, after a decomposition of the initial data into a right-going and a left-going part $c_{0}=c_{0}^{+}+c_{0}^{-}$(strongly depending on the initial time derivative $d_{0}$, see Section 3 for details), let us now define the $\varepsilon$-independent linearized Korteweg-de-Vries (lKdV) equations as follows. 


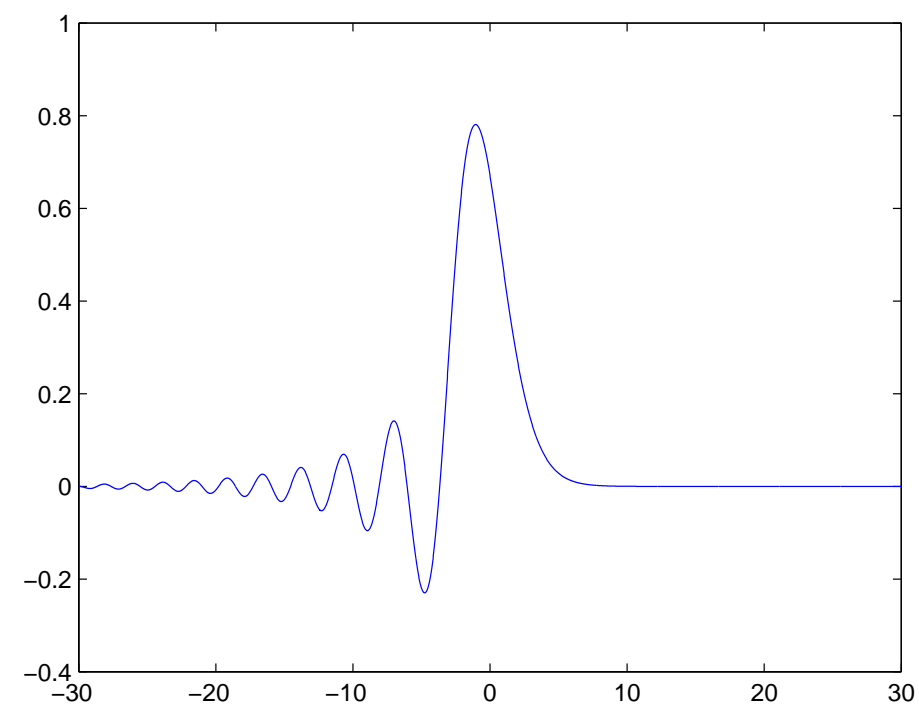

Figure 1: Numerical solution $W^{+}$to the right going $1 \mathrm{KdV}$-problem, evaluated in $t=1$. It is obtained with a finite difference scheme for initial data $W^{+}(x, 0)=\operatorname{sech}(x)=$ $\frac{2}{e^{x}+e^{-x}}$. The solution $W^{-}$is obtained by symmetry.

Definition 2.3 (The $\mathrm{lKdV}$ equations). We distinguish between a right moving and a left moving wave. Denote by $W^{ \pm}(x, t)$ the unique solution to

$$
\begin{aligned}
\partial_{t} W^{ \pm}(x, t) & \pm \frac{a_{2}^{*}}{2 c^{*}} \partial_{x}^{3} W^{ \pm}(x, t)=0, \\
W^{ \pm}(x, 0) & =c_{0}^{ \pm}(x)
\end{aligned}
$$

for $(x, t) \in \mathbb{R} \times(0, T)$.

The existence and uniqueness of a solution is a direct consequence of the results in [10], see Section 3 for more details. We are now able to state the two main results of the paper. The first shows that the weakly dispersive problem of Definition 2.2 provides a good approximation of the original problem.

Theorem 1. Let $c_{0}, d_{0} \in C_{c}^{\infty}((-R, R))$. Consider $u_{\varepsilon}$ from the time-scaled homogenization problem in Definition 2.1 and the weakly dispersive solution $v_{\varepsilon}$ of Definition 2.2. Then the following convergence holds

$$
\left\|u_{\varepsilon}(x, t)-v_{\varepsilon}(x, t)\right\|_{L^{\infty}\left(0, T ; L^{\infty}(\mathbb{R})\right)} \rightarrow 0 \text { for } \varepsilon \rightarrow 0 .
$$

We note that in (4.6),(4.7) a slightly stronger convergence is derived.

The weakly dispersive solution and the $\mathrm{KKdV}$-solution can be compared as follows.

Theorem 2. Consider the weakly dispersive solution $v_{\varepsilon}$ of Definition 2.2 and the shifts $w_{\varepsilon}^{ \pm}$of the lKdV-solutions of Definition 2.3

$$
w_{\varepsilon}^{+}(x, t):=W^{+}\left(x-\frac{c^{*}}{\varepsilon^{2}} t, t\right),
$$




$$
w_{\varepsilon}^{-}(x, t):=W^{-}\left(x+\frac{c^{*}}{\varepsilon^{2}} t, t\right)
$$

Then there exists an $\varepsilon$-independent constant $C$ such that

$$
\left\|\partial_{x} v_{\varepsilon}(x, t)-\partial_{x}\left(w_{\varepsilon}^{+}+w_{\varepsilon}^{-}\right)(x, t)\right\|_{L^{\infty}\left(0, T ; L^{2}(\mathbb{R})\right)} \leq C \varepsilon^{2} .
$$

Moreover,

$$
\left\|v_{\varepsilon}(x, t)-\left(w_{\varepsilon}^{+}+w_{\varepsilon}^{-}\right)(x, t)\right\|_{L^{\infty}\left(0, T ; L^{\infty}(\mathbb{R})\right)} \rightarrow 0 \text { for } \varepsilon \rightarrow 0
$$

Theorem 2 is shown in Section 3. It's proof is rather elementary. By contrast, the proof of Theorem 1 requires more computationally intensive methods and is performed in the last section. By applying the triangle inequality to (2.1) and (2.5) one directly obtains the following result.

Corollary 1. Consider $u_{\varepsilon}$ from the time-scaled homogenization problem and the $l K d V$-solutions $W^{ \pm}$. Then the following convergence holds

$$
\left\|u_{\varepsilon}(x, t)-W^{+}\left(x-\frac{c^{*}}{\varepsilon^{2}} t, t\right)-W^{-}\left(x+\frac{c^{*}}{\varepsilon^{2}} t, t\right)\right\|_{L^{\infty}\left(0, T ; L^{\infty}(\mathbb{R})\right)} \rightarrow 0 \text { for } \varepsilon \rightarrow 0 .
$$

The corollary suggests that the solution to the time-scaled homogenization problem of Definition 2.1 is approximatively equal to two waves propagating with speed $c^{*} / \varepsilon^{2}$ in opposite directions. Moreover, it shows that the shape of the right going wave is well described by the solution $W^{+}$to the $1 \mathrm{KdV}$-problem, those of the left going wave by $W^{-}$.

\section{$3 \quad$ Weakly dispersive equation and $\mathrm{lKdV}$-problem}

This section is devoted to the proof of Theorem 2 .

\subsection{The lKdV-problems and their shifts}

Let us start with some preliminaries concerning the left going and the right going part of the initial data. In fact, due to classical theory, each solution to the one-dimensional wave equation

$$
\partial_{t}^{2} u(x, t)-\left(c^{*}\right)^{2} \partial_{x}^{2} u(x, t)=0
$$

is given by

$$
u(x, t)=f\left(x-c^{*} t\right)+g\left(x+c^{*} t\right) .
$$

In particular,

$$
u(x, 0)=f(x)+g(x) \quad \text { and } \quad \partial_{t} u(x, 0)=-c^{*} \partial_{x} f(x)+c^{*} \partial_{x} g(x) .
$$

Consequently, the following definition is useful. 
Definition 3.1 (Decomposition of the initial data). Let $c^{*}>0$. We define $P_{c^{*}}^{+}\left(c_{0}, d_{0}\right)$ and $P_{c^{*}}^{-}\left(c_{0}, d_{0}\right)$ as the solutions of

$$
\begin{array}{r}
\left(P_{c^{*}}^{+}\left(c_{0}, d_{0}\right)\right)(x)+\left(P_{c^{*}}^{-}\left(c_{0}, d_{0}\right)\right)(x)=c_{0}(x), \\
-c^{*} \partial_{x}\left(P_{c^{*}}^{+}\left(c_{0}, d_{0}\right)\right)(x)+c^{*} \partial_{x}\left(P_{c^{*}}^{-}\left(c_{0}, d_{0}\right)\right)(x)=d_{0}(x) .
\end{array}
$$

In particular, in the case of smooth initial data, $c_{0}, d_{0} \in C_{c}^{\infty}((-R, R))$, also their projections are smooth, i.e. $c_{0}^{+}:=P_{c^{*}}^{+}\left(c_{0}, d_{0}\right), c_{0}^{-}:=P_{c^{*}}^{-}\left(c_{0}, d_{0}\right) \in C_{c}^{\infty}((-R, R))$.

Let us remark that in our case the wave speed as well as the initial data are rescaled, i.e. we consider the wave speed $c^{*} / \varepsilon^{2}$ and initial data $\left(c_{0}, d_{0} / \varepsilon^{2}\right)$. Nevertheless, a simple calculation shows that $P_{c^{*} / \varepsilon^{2}}^{ \pm}\left(c_{0}, d_{0} / \varepsilon^{2}\right)=P_{c^{*}}^{ \pm}\left(c_{0}, d_{0}\right)$.

Next, let us discuss existence and properties of the $1 \mathrm{KdV}$-solution $W^{+}(x, t)$. We omit the analysis of left moving initial data $c_{0}^{-}=P_{c^{*}}^{-}\left(c_{0}, d_{0}\right)$ for convenience, since it can be handled in exactly the same way.

Proposition 3.2 (Existence of the $1 \mathrm{KdV}$-solution and its shift on $\mathbb{R}$ ). Let $c_{0}^{+} \in$ $C_{c}^{\infty}((-R, R))$. Consider the right going lKdV-problem of Definition 2.3. Then,

(i) there exists a unique solution $W^{+}$and a constant $C$ such that

$$
\begin{aligned}
\left\|\partial_{t} \partial_{x}^{3} W^{+}(x, t)\right\|_{L^{\infty}\left(0, T ; L^{2}(\mathbb{R})\right)}^{2} & \leq C\left\|c_{0}^{+}(x)\right\|_{H^{6}((-R, R))}^{2} \\
\left\|\partial_{t}^{2} \partial_{x}^{2} W^{+}(x, t)\right\|_{L^{\infty}\left(0, T ; L^{2}(\mathbb{R})\right)}^{2} & \leq C\left\|c_{0}^{+}(x)\right\|_{H^{9}((-R, R))}^{2}
\end{aligned}
$$

(ii) The shifted function $w_{\varepsilon}^{+}$of (2.2) satisfies $w_{\varepsilon}^{+}, \partial_{t} w_{\varepsilon}^{+}, \partial_{t}^{2} w_{\varepsilon}^{+} \in L^{\infty}\left(0, T ; H^{2}(\mathbb{R})\right)$.

Here, $H^{n}((-R, R))$ as usually denotes the Sobolev-space $H^{n}((-R, R)):=\left\{u(x) \in L^{2}((-R, R))\right.$ with $\partial_{x}^{i} u(x) \in L^{2}((-R, R))$ for $\left.i=1, \ldots, n\right\}$ equipped with the norm $\|u\|_{H^{n}((-R, R))}:=\sum_{i=0}^{n}\left\|\partial_{x}^{i} u\right\|_{L^{2}((-R, R))}$.

Moreover, $H^{0}((-R, R)):=L^{2}((-R, R))$.

Proof. The proof consists of two steps.

First step. In the first step we show the existence of a solution $W^{+}$on bounded intervals $\Omega=(-L, L)$.

Claim 1. On a fixed bounded domain $\Omega=(-L, L)$ with $L>R$, the $1 \mathrm{KdV}$-problem with boundary condition

$$
W^{+}(-L, t)=W^{+}(L, t)=\partial_{x} W^{+}(L, t)=0
$$

has a unique classical solution $W^{+}$. Moreover, there exists an $\Omega$-independent constant $C$, such that

$$
\begin{aligned}
\left\|\partial_{t} \partial_{x}^{3} W^{+}(x, t)\right\|_{L^{\infty}\left(0, T ; L^{2}(\Omega)\right)}^{2} & \leq C\left\|c_{0}^{+}(x)\right\|_{H^{6}(\Omega)}^{2}, \\
\left\|\partial_{t}^{2} \partial_{x}^{2} W^{+}(x, t)\right\|_{L^{\infty}\left(0, T ; L^{2}(\Omega)\right)}^{2} & \leq C\left\|c_{0}^{+}(x)\right\|_{H^{9}(\Omega)}^{2} .
\end{aligned}
$$


A proof of existence and uniqueness of a classical solution can be found in [10]. Let us merely remark that fixing the spatial derivative of $W^{+}$at the right endpoint of the interval is necessary to ensure the well-posedness of the problem.

We have to show the energy estimates in (3.2) and (3.3). Actually, multiplying the $\mathrm{IKdV}$-equation by $W^{+}(x, t)$, integrating over $\Omega$ and applying integration by parts leads to

$$
\begin{aligned}
0 & =\frac{1}{2} \frac{d}{d t}\left\|W^{+}(\cdot, t)\right\|_{L^{2}(\Omega)}^{2}-\frac{a_{2}^{*}}{2 c^{*}} \int_{\Omega} \frac{1}{2} \frac{d}{d x}\left(\partial_{x} W^{+}\right)^{2}(x, t) d x \\
& =\frac{1}{2} \frac{d}{d t}\left\|W^{+}(\cdot, t)\right\|_{L^{2}(\Omega)}^{2}-\frac{a_{2}^{*}}{4 c^{*}}\left(\left(\partial_{x} W^{+}\right)^{2}(L, t)-\left(\partial_{x} W^{+}\right)^{2}(-L, t)\right) \\
& =\frac{1}{2} \frac{d}{d t}\left\|W^{+}(\cdot, t)\right\|_{L^{2}(\Omega)}^{2}+\frac{a_{2}^{*}}{4 c^{*}}\left(\partial_{x} W^{+}\right)^{2}(-L, t) \\
& \geq \frac{1}{2} \frac{d}{d t}\left\|W^{+}(\cdot, t)\right\|_{L^{2}(\Omega)}^{2}
\end{aligned}
$$

and thus for each $t \in(0, T)$

$$
\left\|W^{+}(\cdot, t)\right\|_{L^{2}(\Omega)}^{2} \leq\left\|W^{+}(\cdot, 0)\right\|_{L^{2}(\Omega)}^{2}=\left\|c_{0}^{+}(x)\right\|_{L^{2}(\Omega)}^{2} .
$$

Next, we differentiate the $\mathrm{lKdV}$-equation twice with respect to $t$ arriving at

$$
\begin{gathered}
\partial_{t}\left(\partial_{t}^{2} W^{+}(x, t)\right)+\frac{a_{2}^{*}}{2 c^{*}} \partial_{x}^{3}\left(\partial_{t}^{2} W^{+}(x, t)\right)=0 \\
\partial_{t}^{2} W^{+}(x, 0)=\frac{\left(a_{2}^{*}\right)^{2}}{4\left(c^{*}\right)^{2}} \partial_{x}^{6} c_{0}^{+}(x)
\end{gathered}
$$

In exactly the same way as above we conclude

$$
\left\|\partial_{t}^{2} W^{+}(\cdot, t)\right\|_{L^{2}(\Omega)}^{2} \leq C\left\|c_{0}^{+}(x)\right\|_{H^{6}(\Omega)}^{2}
$$

and thus, taking into account $\partial_{t}^{2} W^{+}=-\frac{a_{2}^{*}}{2 c^{*}} \partial_{t} \partial_{x}^{3} W^{+}$, estimate (3.2) follows. To obtain (3.3) we differentiate once more with respect to $t$ to find

$$
\left\|\partial_{t}^{2} \partial_{x}^{3} W^{+}(x, t)\right\|_{L^{\infty}\left(0, T ; L^{2}(\mathbb{R})\right)}^{2} \leq C\left\|c_{0}^{+}(x)\right\|_{H^{9}(\Omega)}^{2} .
$$

An interpolation argument provides the control of $\left\|\partial_{t}^{2} \partial_{x}^{2} W^{+}(x, t)\right\|_{L^{\infty}\left(0, T ; L^{2}(\mathbb{R})\right)}^{2}$.

Second step. Since the estimates in $(3.2),(3.3)$ are independent of $\Omega=(-L, L)$, one obtains a solution $W^{+}$to the $1 \mathrm{KdV}$-problem on $\mathbb{R}$ by considering $L \rightarrow \infty$. Then $W^{+}$satisfies the same bounds as its approximations.

\subsection{Equation for $v_{\varepsilon}$ in the moving frame}

In what follows, we use that $v_{\varepsilon}$ from Definition 2.2 has some regularity; a proof of existence and regularity can be found in Section 4.1. To motivate the statement of Theorem 2 , let us firstly decompose the weakly dispersive solution $v_{\varepsilon}=v_{\varepsilon}^{+}+v_{\varepsilon}^{-}$as in 
Definition 3.1. To be more precise, we solve the weakly dispersive problem of Definition 2.2 with initial data that belong to a right going wave,

$$
v_{\varepsilon}^{+}(x, 0)=c_{0}^{+}(x) \quad \text { and } \quad \partial_{t} v_{\varepsilon}^{+}(x, 0)=-\frac{c^{*}}{\varepsilon^{2}} \partial_{x} c_{0}^{+}(x) .
$$

We remark that the analysis of the left going part $v_{\varepsilon}^{-}$is analogous.

Observation 1 (Equation in the moving frame). Let $v_{\varepsilon}^{+}$be the weakly dispersive solution of Definition 2.2 with initial data (3.5). Then the shift

$$
V_{\varepsilon}^{+}(x, t):=v_{\varepsilon}^{+}\left(x+\frac{c^{*} t}{\varepsilon^{2}}, t\right)
$$

satisfies the equation

$$
-2 c^{*} \partial_{x}\left(\partial_{t} V_{\varepsilon}^{+}+\frac{a_{2}^{*}}{2 c^{*}} \partial_{x}^{3} V_{\varepsilon}^{+}\right)=-\varepsilon^{2} \partial_{t}\left(\partial_{t} V_{\varepsilon}^{+}+2 \frac{a_{2}^{*}}{c^{*}} \partial_{x}^{3} V_{\varepsilon}^{+}\right)+\varepsilon^{4} \frac{a_{2}^{*}}{a^{*}} \partial_{t}^{2} \partial_{x}^{2} V_{\varepsilon}^{+}
$$

Proof. Due to $v_{\varepsilon}^{+}(x, t)=V_{\varepsilon}^{+}\left(x-\frac{c^{*} t}{\varepsilon^{2}}, t\right)$ one obtains

$$
\begin{aligned}
& \partial_{x} v_{\varepsilon}^{+}=\partial_{x} V_{\varepsilon}^{+} \\
& \partial_{t} v_{\varepsilon}^{+}=-\frac{c^{*}}{\varepsilon^{2}} \partial_{x} V_{\varepsilon}^{+}+\partial_{t} V_{\varepsilon}^{+} \\
& \partial_{t}^{2} v_{\varepsilon}^{+}=\frac{a^{*}}{\varepsilon^{4}} \partial_{x}^{2} V_{\varepsilon}^{+}-\frac{2 c^{*}}{\varepsilon^{2}} \partial_{t} \partial_{x} V_{\varepsilon}^{+}+\partial_{t}^{2} V_{\varepsilon}^{+}
\end{aligned}
$$

A direct calculation now gives the result, using $c^{*} / a^{*}=1 / c^{*}$. In what follows, we use in (1) that $v_{\varepsilon}$ solves the weakly dispersive problem, equality (2) is a direct consequence of the chain rule (3.7)-(3.9).

$$
\begin{aligned}
0 \stackrel{(1)}{=} & \varepsilon^{4} \partial_{t}^{2} v_{\varepsilon}^{+}-a^{*} \partial_{x}^{2} v_{\varepsilon}^{+}-\varepsilon^{6} \frac{a_{2}^{*}}{a^{*}} \partial_{t}^{2} \partial_{x}^{2} v_{\varepsilon}^{+} \\
\stackrel{(2)}{=} & a^{*} \partial_{x}^{2} V_{\varepsilon}^{+}-2 \varepsilon^{2} c^{*} \partial_{t} \partial_{x} V_{\varepsilon}^{+}+\varepsilon^{4} \partial_{t}^{2} V_{\varepsilon}^{+}-a^{*} \partial_{x}^{2} V_{\varepsilon}^{+} \\
& \quad-\varepsilon^{2} a_{2}^{*} \partial_{x}^{4} V_{\varepsilon}^{+}+\frac{2 \varepsilon^{4} a_{2}^{*}}{c^{*}} \partial_{t} \partial_{x}^{3} V_{\varepsilon}^{+}-\frac{\varepsilon^{6} a_{2}^{*}}{a^{*}} \partial_{x}^{2} \partial_{t}^{2} V_{\varepsilon}^{+} \\
= & \varepsilon^{2}\left(-2 c^{*} \partial_{t} \partial_{x} V_{\varepsilon}^{+}-a_{2}^{*} \partial_{x}^{4} V_{\varepsilon}^{+}+\varepsilon^{2} \partial_{t}^{2} V_{\varepsilon}^{+}+\frac{2 \varepsilon^{2} a_{2}^{*}}{c^{*}} \partial_{t} \partial_{x}^{3} V_{\varepsilon}^{+}-\frac{\varepsilon^{4} a_{2}^{*}}{a^{*}} \partial_{x}^{2} \partial_{t}^{2} V_{\varepsilon}^{+}\right)
\end{aligned}
$$

and hence (3.6).

Loosely speaking, Observation 1 suggests that the shift $V_{\varepsilon}^{+}$satisfies the $\mathrm{lKdV}$ equation

$$
\partial_{t} V_{\varepsilon}^{+}(x, t)+\frac{a_{2}^{*}}{2 c^{*}} \partial_{x}^{3} V_{\varepsilon}^{+}(x, t)=O\left(\varepsilon^{2}\right)
$$

and can therefore be compared to leading order with $W^{+}(x, t)$. In order to make the connection precise, we now start from the $\mathrm{KKdV}$-solution $W^{+}(x, t)$ and derive a corresponding equation for $w_{\varepsilon}^{+}(x, t)$. 
Lemma 3.3. Consider the lKdV-solution $W^{+}(x, t)$ of Definition 2.3 and its left shift $w_{\varepsilon}^{+}(x, t)=W^{+}\left(x-\frac{c^{*} t}{\varepsilon^{2}}, t\right)$. Then $w_{\varepsilon}^{+}(x, t)$ solves the following problem

$$
\begin{aligned}
\varepsilon^{4} \partial_{t}^{2} w_{\varepsilon}^{+}(x, t)-a^{*} \partial_{x}^{2} w_{\varepsilon}^{+}(x, t)-\varepsilon^{6} \frac{a_{2}^{*}}{a^{*}} \partial_{t}^{2} \partial_{x}^{2} w_{\varepsilon}^{+}(x, t) \\
=-\varepsilon^{6} \frac{a_{2}^{*}}{a^{*}} \partial_{t}^{2} \partial_{x}^{2} W^{+}\left(x-\frac{c^{*}}{\varepsilon^{2}} t, t\right)+\varepsilon^{4} \frac{3 a_{2}^{*}}{2 c^{*}} \partial_{x}^{3} \partial_{t} W^{+}\left(x-\frac{c^{*}}{\varepsilon^{2}} t, t\right), \\
w_{\varepsilon}^{+}(x, 0)=c_{0}^{+}(x), \\
\partial_{t} w_{\varepsilon}^{+}(x, 0)=-\frac{c^{*}}{\varepsilon^{2}} \partial_{x} c_{0}^{+}(x)-\frac{a_{2}^{*}}{2 c^{*}} \partial_{x}^{3} c_{0}^{+}(x) .
\end{aligned}
$$

Proof. As in Observation 1, omitting the arguments $(x, t)$ for convenience, the chain rule yields

$$
\begin{aligned}
\partial_{x} W^{+} & =\partial_{x} w_{\varepsilon}^{+} \\
\partial_{t} W^{+} & =\frac{c^{*}}{\varepsilon^{2}} \partial_{x} w_{\varepsilon}^{+}+\partial_{t} w_{\varepsilon}^{+} \\
\partial_{t}^{2} W^{+} & =\frac{a^{*}}{\varepsilon^{4}} \partial_{x}^{2} w_{\varepsilon}^{+}+\frac{2 c^{*}}{\varepsilon^{2}} \partial_{t} \partial_{x} w_{\varepsilon}^{+}+\partial_{t}^{2} w_{\varepsilon}^{+}
\end{aligned}
$$

and thus

$$
0=\partial_{t} W^{+}+\frac{a_{2}^{*}}{2 c^{*}} \partial_{x}^{3} W^{+}=\frac{c^{*}}{\varepsilon^{2}} \partial_{x} w_{\varepsilon}^{+}+\partial_{t} w_{\varepsilon}^{+}+\frac{a_{2}^{*}}{2 c^{*}} \partial_{x}^{3} w_{\varepsilon}^{+}
$$

Now, we apply the partial differential operator $\varepsilon^{4} \partial_{t}-\varepsilon^{2} c^{*} \partial_{x}$ to (3.11). Inserting the term $\varepsilon^{6} \frac{a_{2}^{*}}{a^{*}} \partial_{t}^{2} \partial_{x}^{2} w_{\varepsilon}^{+}$and observing that the expression $\varepsilon^{2} c^{*} \partial_{t} \partial_{x} w_{\varepsilon}^{+}$appears twice with different sign, one arrives at

$$
\begin{aligned}
& 0= \varepsilon^{4} \partial_{t}^{2} w_{\varepsilon}^{+}-a^{*} \partial_{x}^{2} w_{\varepsilon}^{+}-\varepsilon^{6} \frac{a_{2}^{*}}{a^{*}} \partial_{t}^{2} \partial_{x}^{2} w_{\varepsilon}^{+} \\
&+\varepsilon^{6} \frac{a_{2}^{*}}{a^{*}} \partial_{t}^{2} \partial_{x}^{2} w_{\varepsilon}^{+}+\varepsilon^{2} \frac{a_{2}^{*}}{2 c^{*}}\left(\varepsilon^{2} \partial_{t} \partial_{x}^{3} w_{\varepsilon}^{+}-c^{*} \partial_{x}^{4} w_{\varepsilon}^{+}\right) \\
& \stackrel{(3.10)}{=} \varepsilon^{4} \partial_{t}^{2} w_{\varepsilon}^{+}-a^{*} \partial_{x}^{2} w_{\varepsilon}^{+}-\varepsilon^{6} \frac{a_{2}^{*}}{a^{*}} \partial_{t}^{2} \partial_{x}^{2} w_{\varepsilon}^{+} \\
&+\frac{a_{2}^{*}}{a^{*}} \varepsilon^{6} \partial_{x}^{2} \partial_{t}^{2} W^{+}-\varepsilon^{4} \frac{3 a_{2}^{*}}{2 c^{*}}\left(\frac{c^{*}}{\varepsilon^{2}} \partial_{x}^{4} w_{\varepsilon}^{+}+\partial_{x}^{3} \partial_{t} w^{+}\right) \\
& \stackrel{(3.10)}{=} \varepsilon^{4} \partial_{t}^{2} w_{\varepsilon}^{+}-a^{*} \partial_{x}^{2} w_{\varepsilon}^{+}-\varepsilon^{6} \frac{a_{2}^{*}}{a^{*}} \partial_{t}^{2} \partial_{x}^{2} w_{\varepsilon}^{+} \\
&+\varepsilon^{6} \frac{a_{2}^{*}}{a^{*}} \partial_{x}^{2} \partial_{t}^{2} W^{+}-\varepsilon^{4} \frac{3 a_{2}^{*}}{2 c^{*}} \partial_{x}^{3} \partial_{t} W^{+},
\end{aligned}
$$

which is the claimed result. It remains to verify the initial conditions. Actually,

$$
-\frac{a_{2}^{*}}{2 c^{*}} \partial_{x}^{3} W^{+}(x, 0)=\partial_{t} W^{+}(x, 0)=\frac{c^{*}}{\varepsilon^{2}} \partial_{x} w_{\varepsilon}^{+}(x, 0)+\partial_{t} w_{\varepsilon}^{+}(x, 0) .
$$


Taking into account

$$
\begin{aligned}
-\frac{a_{2}^{*}}{2 c^{*}} \partial_{x}^{3} W^{+}(x, 0) & =-\frac{a_{2}^{*}}{2 c^{*}} \partial_{x}^{3} c_{0}^{+}(x), \\
\frac{c^{*}}{\varepsilon^{2}} \partial_{x} w_{\varepsilon}^{+}(x, 0) & =\frac{c^{*}}{\varepsilon^{2}} \partial_{x} c_{0}^{+}(x),
\end{aligned}
$$

one finally arrives at

$$
\partial_{t} w_{\varepsilon}^{+}(x, 0)=-\frac{a_{2}^{*}}{2 c^{*}} \partial_{x}^{3} c_{0}^{+}(x)-\frac{c^{*}}{\varepsilon^{2}} \partial_{x} c_{0}^{+}(x)
$$

This shows the equation for $w_{\varepsilon}^{+}$and concludes the proof.

\subsection{Proof of Theorem 2}

We are now in the position to prove Theorem 2. The main estimate is contained in Proposition 3.4 below for right moving initial data. We will observe later that Theorem 2 can easily be derived from that proposition. In what follows, $C$ denotes different $\varepsilon$-independent constants. We will use the following integration by parts formula for $H^{1}(\mathbb{R})$-functions.

Claim 2 (Integration by parts). Let $f(x), g(x) \in H^{1}(\mathbb{R})$. Then

$$
\int_{\mathbb{R}} \partial_{x} f(x) g(x) d x=-\int_{\mathbb{R}} f(x) \partial_{x} g(x) d x .
$$

This result is easily obtained by approximating the function $f(x) \in H^{1}(\mathbb{R})$ with $\phi_{k}(x) \in C_{c}^{\infty}(\mathbb{R})$ such that $\left\|f-\phi_{k}\right\|_{H^{1}(\mathbb{R})} \rightarrow 0$.

Proposition 3.4. Let $v_{\varepsilon}^{+}$be the solution to the weakly dispersive problem of Definition 2.2 with initial data (3.5). Let $W^{+}$be the solution to the right going lKdV-problem of Definition 2.3 and let $w_{\varepsilon}^{+}$be its shift, $w_{\varepsilon}^{+}(x, t)=W^{+}\left(x-\frac{c^{*} t}{\varepsilon^{2}}, t\right)$. Then there exists an $\varepsilon$-independent constant $C$, such that

$$
\begin{aligned}
\left\|\partial_{x} v_{\varepsilon}^{+}-\partial_{x} w_{\varepsilon}^{+}\right\|_{L^{\infty}\left(0, T ; L^{2}(\mathbb{R})\right)} & \leq C \varepsilon^{2} \\
\left\|v_{\varepsilon}^{+}-w_{\varepsilon}^{+}\right\|_{L^{\infty}\left(0, T ; L^{2}(\mathbb{R})\right)} & \leq C
\end{aligned}
$$

Proof. Since $v_{\varepsilon}^{+}$solves the weakly dispersive equation, Lemma 3.3 yields that the difference $w_{\varepsilon}^{+}-v_{\varepsilon}^{+}$satisfies

$$
\begin{aligned}
\varepsilon^{4} \partial_{t}^{2}\left(w_{\varepsilon}^{+}\right. & \left.-v_{\varepsilon}^{+}\right)(x, t)-a^{*} \partial_{x}^{2}\left(w_{\varepsilon}^{+}-v_{\varepsilon}^{+}\right)(x, t)-\varepsilon^{6} \frac{a_{2}^{*}}{a^{*}} \partial_{t}^{2} \partial_{x}^{2}\left(w_{\varepsilon}^{+}-v_{\varepsilon}^{+}\right)(x, t) \\
& =-\varepsilon^{6} \frac{a_{2}^{*}}{a^{*}} \partial_{t}^{2} \partial_{x}^{2} W^{+}\left(x-\frac{c^{*}}{\varepsilon^{2}} t, t\right)+\varepsilon^{4} \frac{3 a_{2}^{*}}{2 c^{*}} \partial_{x}^{3} \partial_{t} W^{+}\left(x-\frac{c^{*}}{\varepsilon^{2}} t, t\right) .
\end{aligned}
$$


Now, we multiply $(3.15)$ by $\partial_{t}\left(w_{\varepsilon}^{+}-v_{\varepsilon}^{+}\right)$and integrate over $\mathbb{R}$. According to (3.12), we then apply integration by parts to obtain

$$
\begin{aligned}
\frac{1}{2} & \frac{d}{d t}\left(\varepsilon^{4}\left\|\partial_{t} w_{\varepsilon}^{+}(\cdot, t)-\partial_{t} v_{\varepsilon}^{+}(\cdot, t)\right\|_{L^{2}(\mathbb{R})}^{2}+a^{*}\left\|\partial_{x} w_{\varepsilon}^{+}(\cdot, t)-\partial_{x} v_{\varepsilon}^{+}(\cdot, t)\right\|_{L^{2}(\mathbb{R})}^{2}\right. \\
& \left.+\varepsilon^{6} \frac{a_{2}^{*}}{a^{*}}\left\|\partial_{t} \partial_{x} w_{\varepsilon}^{+}(\cdot, t)-\partial_{t} \partial_{x} v_{\varepsilon}^{+}(\cdot, t)\right\|_{L^{2}(\mathbb{R})}^{2}\right) \\
= & -\int_{\mathbb{R}}\left(\varepsilon^{6} \frac{a_{2}^{*}}{a^{*}} \partial_{t}^{2} \partial_{x}^{2} W^{+}+\varepsilon^{4} \frac{3 a_{2}^{*}}{2 c^{*}} \partial_{x}^{3} \partial_{t} W^{+}\right)\left(x-\frac{c^{*}}{\varepsilon^{2}} t, t\right)\left(\partial_{t} w_{\varepsilon}^{+}-\partial_{t} v_{\varepsilon}^{+}\right)(x, t) d x \\
\leq & \frac{4}{\varepsilon^{4}}\left(\left\|\varepsilon^{6} \frac{a_{2}^{*}}{a^{*}} \partial_{t}^{2} \partial_{x}^{2} W^{+}\left(\cdot-\frac{c^{*}}{\varepsilon^{2}} t, t\right)\right\|_{L^{2}(\mathbb{R})}^{2}+\left\|\varepsilon^{4} \frac{3 a_{2}^{*}}{2 c^{*}} \partial_{x}^{3} \partial_{t} W^{+}\left(\cdot-\frac{c^{*}}{\varepsilon^{2}} t, t\right)\right\|_{L^{2}(\mathbb{R})}^{2}\right) \\
& \left.+\frac{\varepsilon^{4}}{2}\left\|\partial_{t} w_{\varepsilon}^{+}(\cdot, t)-\partial_{t} v_{\varepsilon}^{+}(\cdot, t)\right\|_{L^{2}(\mathbb{R})}^{2}\right) \\
= & 4\left(\varepsilon^{8}\left\|\frac{a_{2}^{*}}{a^{*}} \partial_{t}^{2} \partial_{x}^{2} W^{+}\left(\cdot-\frac{c^{*}}{\varepsilon^{2}} t, t\right)\right\|_{L^{2}(\mathbb{R})}^{2}+\varepsilon^{4}\left\|\frac{3 a_{2}^{*}}{2 c^{*}} \partial_{x}^{3} \partial_{t} W^{+}\left(\cdot-\frac{c^{*}}{\varepsilon^{2}} t, t\right)\right\|_{L^{2}(\mathbb{R})}^{2}\right) \\
& +\frac{\varepsilon^{4}}{2}\left\|\partial_{t} w_{\varepsilon}^{+}(\cdot, t)-\partial_{t} v_{\varepsilon}^{+}(\cdot, t)\right\|_{L^{2}(\mathbb{R})}^{2}
\end{aligned}
$$

for almost every $t \in[0, T]$. Next, we define

$$
\begin{aligned}
A(t):= & \frac{1}{2}\left(\varepsilon^{4}\left\|\partial_{t} w_{\varepsilon}^{+}(\cdot, t)-\partial_{t} v_{\varepsilon}^{+}(\cdot, t)\right\|_{L^{2}(\mathbb{R})}^{2}+a^{*}\left\|\partial_{x} w_{\varepsilon}^{+}(\cdot, t)-\partial_{x} v_{\varepsilon}^{+}(\cdot, t)\right\|_{L^{2}(\mathbb{R})}^{2}\right. \\
& \left.+\varepsilon^{6} \frac{a_{2}^{*}}{a^{*}}\left\|\partial_{t} \partial_{x} w_{\varepsilon}^{+}(\cdot, t)-\partial_{t} \partial_{x} v_{\varepsilon}^{+}(\cdot, t)\right\|_{L^{2}(\mathbb{R})}^{2}\right) .
\end{aligned}
$$

Taking into account

$$
\left(w_{\varepsilon}^{+}-v_{\varepsilon}^{+}\right)(x, 0)=0 \quad \text { and } \quad \partial_{t}\left(w_{\varepsilon}^{+}-v_{\varepsilon}^{+}\right)(x, 0)=-\frac{a_{2}^{*}}{2 c^{*}} \partial_{x}^{3} c_{0}^{+}(x)
$$

and applying the Gronwall Lemma finally leads to

$$
\begin{aligned}
A(t) \leq & C\left(\frac{\varepsilon^{4}}{2}\left\|\frac{a_{2}^{*}}{2 c^{*}} \partial_{x}^{3} c_{0}^{+}(x)\right\|_{L^{2}(\mathbb{R})}^{2}+\frac{\varepsilon^{6} a_{2}^{*}}{2 a^{*}}\left\|\frac{a_{2}^{*}}{2 c^{*}} \partial_{x}^{4} c_{0}^{+}(x)\right\|_{L^{2}(\mathbb{R})}^{2}\right. \\
& \left.+\varepsilon^{8}\left\|\frac{a_{2}^{*}}{a^{*}} \partial_{t}^{2} \partial_{x}^{2} W^{+}\left(x-\frac{c^{*}}{\varepsilon^{2}} t, t\right)\right\|_{L^{2}\left(0, T ; L^{2}(\mathbb{R})\right)}^{2}\right) \\
& \left.+\varepsilon^{4}\left\|\frac{3 a_{2}^{*}}{2 c^{*}} \partial_{x}^{3} \partial_{t} W^{+}\left(x-\frac{c^{*}}{\varepsilon^{2}} t, t\right)\right\|_{L^{2}\left(0, T ; L^{2}(\mathbb{R})\right)}^{2}\right) \\
\leq & C\left(\varepsilon^{4}\left\|c_{0}^{+}(x)\right\|_{H^{4}((-R, R))}^{2}+\varepsilon^{8}\left\|\partial_{t}^{2} \partial_{x}^{2} W^{+}\left(x-\frac{c^{*}}{\varepsilon^{2}} t, t\right)\right\|_{L^{2}\left(0, T ; L^{2}(\mathbb{R})\right)}^{2}\right. \\
& \left.+\varepsilon^{4}\left\|\partial_{x}^{3} \partial_{t} W^{+}\left(x-\frac{c^{*}}{\varepsilon^{2}} t, t\right)\right\|_{L^{2}\left(0, T ; L^{2}(\mathbb{R})\right)}^{2}\right) \\
\stackrel{(1)}{\leq} & C \varepsilon^{4}\left\|c_{0}^{+}(x)\right\|_{H^{9}((-R, R))}^{2} \leq C \varepsilon^{4} .
\end{aligned}
$$


for almost every $t \in[0, T]$. Let us remark that inequality (1) is a consequence of Proposition 3.2. This shows (3.13) and that

$$
\left\|\partial_{t} w_{\varepsilon}^{+}-\partial_{t} v_{\varepsilon}^{+}\right\|_{L^{\infty}\left(0, T ; L^{2}(\mathbb{R})\right)} \leq C
$$

Finally, to prove inequality (3.14), one simply writes

$$
\begin{aligned}
\left(w_{\varepsilon}^{+}-v_{\varepsilon}^{+}\right)(x, t) & =\left(w_{\varepsilon}^{+}-v_{\varepsilon}^{+}\right)(x, 0)+\int_{0}^{t}\left(\partial_{t} w_{\varepsilon}^{+}-\partial_{t} v_{\varepsilon}^{+}\right)(x, \tau) d \tau \\
& =\int_{0}^{t}\left(\partial_{t} w_{\varepsilon}^{+}-\partial_{t} v_{\varepsilon}^{+}\right)(x, \tau) d \tau
\end{aligned}
$$

and applies (3.16).

In exactly the same way the analogous result can be stated for the left going weakly dispersive solution $v_{\varepsilon}^{-}$and its shift $w_{\varepsilon}^{-}(x, t):=W^{-}\left(x+\frac{c^{*} t}{\varepsilon^{2}}, t\right)$. Due to $v_{\varepsilon}=v_{\varepsilon}^{+}+v_{\varepsilon}^{-}$ and the linearity of the weakly dispersive problem one obtains

Proposition 3.5. Let $v_{\varepsilon}$ be the weakly dispersive solution of Definition 2.2 and let $w_{\varepsilon}^{+}, w_{\varepsilon}^{-}$be as in Theorem 2. Then the following estimates are valid

$$
\begin{gathered}
\left\|\partial_{x} v_{\varepsilon}-\partial_{x}\left(w_{\varepsilon}^{+}+w_{\varepsilon}^{-}\right)\right\|_{L^{\infty}\left(0, T ; L^{2}(\mathbb{R})\right)} \leq C \varepsilon^{2} \\
\left\|v_{\varepsilon}-\left(w_{\varepsilon}^{+}+w_{\varepsilon}^{-}\right)\right\|_{L^{\infty}\left(0, T ; L^{2}(\mathbb{R})\right)} \leq C .
\end{gathered}
$$

We are now in the position to prove Theorem 2 .

Proof of Theorem 2. Inequality (2.4) appears in Proposition 3.5.

We have to prove $(2.5)$, that

$$
\left\|v_{\varepsilon}(x, t)-\left(w_{\varepsilon}^{+}+w_{\varepsilon}^{-}\right)(x, t)\right\|_{L^{\infty}\left(0, T ; L^{\infty}(\mathbb{R})\right)} \rightarrow 0 \text { for } \varepsilon \rightarrow 0
$$

Considering the difference $z_{\varepsilon}(x, t):=\left(v_{\varepsilon}-\left(w_{\varepsilon}^{+}+w_{\varepsilon}^{-}\right)\right)(x, t)$, we claim that

$$
\sup _{t \in[0, T]}\left\|z_{\varepsilon}(\cdot, t)-z_{\varepsilon}(y, t)\right\|_{L^{\infty}(\mathbb{R})} \leq C \varepsilon^{2}
$$

for each $y \in \mathbb{R}$. Actually, due to Proposition 3.5 one obtains

$$
\begin{aligned}
\sup _{t \in[0, T]}\left\|z_{\varepsilon}(\cdot, t)-z_{\varepsilon}(y, t)\right\|_{L^{\infty}(\mathbb{R})}^{2} & =\sup _{t \in[0, T]}\left\|\left(\int_{y} \partial_{x} z_{\varepsilon}(\xi, t) d \xi\right)\right\|_{L^{\infty}(\mathbb{R})}^{2} \\
& \leq\left\|\partial_{x} z_{\varepsilon}\right\|_{L^{\infty}\left(0, T ; L^{2}(\mathbb{R})\right)}^{2} \leq C \varepsilon^{4}
\end{aligned}
$$

It remains to prove that there exists $y_{0} \in \mathbb{R}$ such that

$$
\sup _{t \in[0, T]}\left|z_{\varepsilon}\left(y_{0}, t\right)\right| \stackrel{\varepsilon \rightarrow 0}{\rightarrow} 0
$$


Then, by applying the triangle inequality, convergence (3.19) follows directly,

$$
\begin{aligned}
\left\|z_{\varepsilon}(x, t)\right\|_{L^{\infty}\left(0, T ; L^{\infty}(\mathbb{R})\right)} & \leq \sup _{t \in[0, T]}\left\|z_{\varepsilon}(\cdot, t)-z_{\varepsilon}\left(y_{0}, t\right)\right\|_{L^{\infty}(\mathbb{R})}+\sup _{t \in[0, T]}\left|z_{\varepsilon}\left(y_{0}, t\right)\right| \\
& \leq C \varepsilon^{2}+\sup _{t \in[0, T]}\left|z_{\varepsilon}\left(y_{0}, t\right)\right| \stackrel{\varepsilon \rightarrow 0}{\rightarrow} 0 .
\end{aligned}
$$

To show (3.21) we assume that, for $y_{0} \in \mathbb{R}$, there exist $\delta>0$ and sequences $\varepsilon_{k} \rightarrow 0$ and $t_{k} \in[0, T]$ such that

$$
\left|z_{\varepsilon_{k}}\left(y_{0}, t_{k}\right)\right|>\delta
$$

Then, due to (3.20) one obtains

$$
\left|z_{\varepsilon_{k}}\left(x, t_{k}\right)\right|>\delta / 2
$$

for every $x \in \mathbb{R}$ and $k$ sufficiently large. This contradicts inequality (3.18) in Proposition 3.5 and thus convergence (3.21) follows.

\section{The original homogenization problem and the weakly dispersive equation}

This section is devoted to the proof of Theorem 1. We will study properties of the weakly dispersive problem in Subsection 4.1. In Subsection 4.2 we discuss the adaption operator $\mathcal{A}_{\varepsilon}$. Finally, in Subsection 4.3 the proof of Theorem 1 is given.

\subsection{The weakly dispersive problem}

In this subsection, we prove existence and uniqueness of a solution to the weakly dispersive problem of Definition 2.2. Besides, energy estimates and regularity properties are discussed. As for the $\mathrm{lKdV}$-problem, we firstly construct solutions on bounded intervals $\Omega=(-L, L)$ and show that the corresponding energy estimates are $\Omega$-independent.

Proposition 4.1 (Existence on $\mathbb{R}$ and energy estimates). Let $k \geq 1$ and $c_{0}, d_{0} \in$ $C_{c}^{\infty}((-R, R))$. Consider the weakly dispersive problem of Definition 2.2. Then there exists a unique solution $v_{\varepsilon}$ such that $v_{\varepsilon} \in L^{\infty}\left(0, T ; H^{k}(\mathbb{R})\right), \partial_{t} v_{\varepsilon} \in L^{\infty}\left(0, T ; H^{k-1}(\mathbb{R})\right)$, $\partial_{t}^{2} v_{\varepsilon} \in L^{2}\left(0, T ; H^{k-1}(\mathbb{R})\right)$. Moreover, the following energy estimates are valid

$$
\begin{aligned}
\left\|\partial_{x} v_{\varepsilon}\right\|_{L^{\infty}\left(0, T ; H^{k-1}(\mathbb{R})\right)} & +\varepsilon^{2}\left\|\partial_{t} v_{\varepsilon}\right\|_{L^{\infty}\left(0, T ; H^{k-1}(\mathbb{R})\right)} \\
& +\varepsilon^{5}\left\|\partial_{t}^{2} v_{\varepsilon}\right\|_{L^{2}\left(0, T ; H^{k-1}(\mathbb{R})\right)} \leq C K_{k}\left(c_{0}, d_{0}\right),
\end{aligned}
$$

where the constant $C$ is independent of $\varepsilon$ and

$K_{k}\left(c_{0}, d_{0}\right):=\left(\left\|\partial_{x} c_{0}\right\|_{H^{k-1}((-R, R))}^{2}+\left\|d_{0}\right\|_{H^{k-1}((-R, R))}^{2}+\varepsilon^{2}\left\|\partial_{x} d_{0}\right\|_{H^{k-1}((-R, R))}^{2}\right)^{1 / 2}$.

Proof. Our aim is to apply the Rothe time discretization method. More precisely, we discretize the time variable by introducing a finite number of time-steps $t_{j}$ and replace the time-derivative by a difference quotient $\partial_{t} v_{\varepsilon}\left(t_{j}\right):=\frac{v_{\varepsilon}\left(t_{j}\right)-v_{\varepsilon}\left(t_{j-1}\right)}{\Delta t}$. In each time-step 
$t_{j}$ we then solve an ordinary differential equation on $\Omega=(-L, L)$ and derive a priori estimates which are independent of the domain $\Omega$. Considering $L \rightarrow \infty$ provides a solution on $\mathbb{R}$ in each time-step. Finally, we consider the limit $\Delta t \rightarrow 0$ in the discretization scheme. In the following we will merely give the proof of the corresponding a priori estimates for $k=1$ and omit the details of the method. Actually, one derives estimates for higher order spatial derivatives by differentiating the weakly dispersive equation with respect to $x$.

First energy estimates. Multiplying the weakly dispersive equation of Definition 2.2 by $\partial_{t} v_{\varepsilon}$ and integrating over $\Omega$, one obtains

$$
\frac{1}{2} \frac{d}{d t}\left(\varepsilon^{4}\left\|\partial_{t} v_{\varepsilon}(\cdot, t)\right\|_{L^{2}(\Omega)}^{2}+a^{*}\left\|\partial_{x} v_{\varepsilon}(\cdot, t)\right\|_{L^{2}(\Omega)}^{2}+\varepsilon^{6} \frac{a_{2}^{*}}{a^{*}}\left\|\partial_{t} \partial_{x} v_{\varepsilon}(\cdot, t)\right\|_{L^{2}(\Omega)}^{2}\right)=0 .
$$

Next, we integrate over $[0, t]$ arriving at

$$
\begin{aligned}
\varepsilon^{4}\left\|\partial_{t} v_{\varepsilon}(\cdot, t)\right\|_{L^{2}(\Omega)}^{2} & +a^{*}\left\|\partial_{x} v_{\varepsilon}(\cdot, t)\right\|_{L^{2}(\Omega)}^{2}+\varepsilon^{6} \frac{a_{2}^{*}}{a^{*}}\left\|\partial_{t} \partial_{x} v_{\varepsilon}(\cdot, t)\right\|_{L^{2}(\Omega)}^{2} \\
& =\varepsilon^{4}\left\|\frac{1}{\varepsilon^{2}} d_{0}(x)\right\|_{L^{2}(\Omega)}^{2}+a^{*}\left\|\partial_{x} c_{0}(x)\right\|_{L^{2}(\Omega)}^{2}+\varepsilon^{6} \frac{a_{2}^{*}}{a^{*}}\left\|\frac{1}{\varepsilon^{2}} \partial_{x} d_{0}(x)\right\|_{L^{2}(\Omega)}^{2} \\
& =\left\|d_{0}(x)\right\|_{L^{2}((-R, R)}^{2}+a^{*}\left\|\partial_{x} c_{0}(x)\right\|_{L^{2}((-R, R))}^{2}+\varepsilon^{2} \frac{a_{2}^{*}}{a^{*}}\left\|\partial_{x} d_{0}(x)\right\|_{L^{2}((-R, R))}^{2} \\
& \leq C\left(K_{1}\left(c_{0}, d_{0}\right)\right)^{2}
\end{aligned}
$$

for each $t \in[0, T]$, which proves the estimates for the first two terms in (4.1).

Second energy estimates. It remains to verify the estimate for $\partial_{t}^{2} v_{\varepsilon}(x, t)$. Actually,

$$
\varepsilon^{4} \partial_{t}^{2} v_{\varepsilon}(x, t)-\varepsilon^{6} \frac{a_{2}^{*}}{a^{*}} \partial_{x}^{2} \partial_{t}^{2} v_{\varepsilon}(x, t)-a^{*} \partial_{x}^{2} v_{\varepsilon}(x, t)=0 .
$$

Multiplying equation (4.2) by $\partial_{t}^{2} v_{\varepsilon}(x, t)$, integrating over $\Omega \times(0, T)$ and applying integration by parts leads to

$$
\begin{aligned}
\varepsilon^{4}\left\|\partial_{t}^{2} v_{\varepsilon}\right\|_{L^{2}\left(0, T ; L^{2}(\Omega)\right)}^{2} & +\varepsilon^{6} \frac{a_{2}^{*}}{a^{*}}\left\|\partial_{t}^{2} \partial_{x} v_{\varepsilon}\right\|_{L^{2}\left(0, T ; L^{2}(\Omega)\right)}^{2} \\
& =-\int_{0}^{T} \int_{\Omega} a^{*} \partial_{x} v_{\varepsilon}(x, t) \partial_{t}^{2} \partial_{x} v_{\varepsilon}(x, t) d x d t \\
& \leq\left\|a^{*} \partial_{x} v_{\varepsilon}\right\|_{L^{2}\left(0, T ; L^{2}(\Omega)\right)}\left\|\partial_{t}^{2} \partial_{x} v_{\varepsilon}\right\|_{L^{2}\left(0, T ; L^{2}(\Omega)\right)} \\
& \leq \varepsilon^{6} \gamma\left\|\partial_{x} \partial_{t}^{2} v_{\varepsilon}\right\|_{L^{2}\left(0, T ; L^{2}(\Omega)\right)}^{2}+\frac{1}{\varepsilon^{6} \gamma}\left\|a^{*} \partial_{x} v_{\varepsilon}\right\|_{L^{2}\left(0, T ; L^{2}(\Omega)\right)}^{2}
\end{aligned}
$$

where we choose $\gamma \in\left(0, \frac{a_{2}^{*}}{a^{*}}\right)$. Hence,

$$
\begin{aligned}
\varepsilon^{4}\left\|\partial_{t}^{2} v_{\varepsilon}\right\|_{L^{2}\left(0, T ; L^{2}(\Omega)\right)}^{2}+\varepsilon^{6}\left\|\partial_{t}^{2} \partial_{x} v_{\varepsilon}\right\|_{L^{2}\left(0, T ; L^{2}(\Omega)\right)}^{2} & \leq \frac{C}{\varepsilon^{6}}\left\|\partial_{x} v_{\varepsilon}\right\|_{L^{2}\left(0, T ; L^{2}(\Omega)\right)}^{2} \\
& \leq \frac{C}{\varepsilon^{6}}\left(K_{1}\left(c_{0}, d_{0}\right)\right)^{2}
\end{aligned}
$$


due to the first energy estimates. Consequently,

$$
\left\|\partial_{t}^{2} v_{\varepsilon}\right\|_{L^{2}\left(0, T ; L^{2}(\Omega)\right)} \leq \frac{1}{\varepsilon^{5}} C K_{1}\left(c_{0}, d_{0}\right)
$$

which is the claimed result.

\subsection{The adaption operator}

The aim of this section is to adapt a function $f(x, t)$ to the micro-structure of the material. More precisely, our aim is to construct an operator $\mathcal{A}_{\varepsilon}$ such that $\partial_{x}\left(a\left(\frac{x}{\varepsilon}\right) \partial_{x}\left(\mathcal{A}_{\varepsilon}(f)\right)\right)$ can be expanded in derivatives of $f$, i.e.

$$
\partial_{x}\left(a\left(\frac{x}{\varepsilon}\right) \partial_{x}\left(\mathcal{A}_{\varepsilon}(f)\right)\right)-\mathcal{A}_{\varepsilon}\left(\sum_{i=0}^{m} \varepsilon^{i} a_{i}^{*} \partial_{x}^{2+i} f\right)=\rho,
$$

where $\rho$ is a small error, $m \in \mathbb{N}$ and $a_{i}^{*} \in \mathbb{R}$. If (4.3) is satisfied, we have a way to replace the elliptic operator with oscillating coefficients by a finite sum of differential operators with constant coefficients.

Let us start with some preliminaries. Considering $Y=(0,1)$ as in the introduction, we denote by $H_{\text {per }}^{1}(Y)$ the closure of $\left\{u(\cdot) \in C^{\infty}(\mathbb{R}) \mid u\right.$ is $Y$-periodic $\}$ with respect to the Sobolev-norm \|\|$_{H^{1}(Y)}$. Moreover, let

$$
\langle u(\cdot)\rangle_{Y}:=\frac{1}{|Y|} \int_{Y} u(y) d y=\int_{Y} u(y) d y
$$

be the mean value of $u(\cdot)$ and let $C_{\text {per }}^{2}(Y):=\left\{L(\cdot) \in C^{2}(\mathbb{R}) \mid L(\cdot)\right.$ is Y-periodic $\}$. Then, formal series expansions in powers of $\varepsilon$ and numerical results (see, for instance, $[6,7])$ suggest that the solution to the time-scaled homogenization problem of Definition 2.1 can be approximated by functions which obey the following general structure. We cut off the expansion after the sixth expansion term.

Definition 4.2 (Adaption operator $\left.\mathcal{A}_{\varepsilon}\right)$. Let $f(\cdot, t) \in H^{8}(\mathbb{R})$ for each $t$ and let $L_{1}(\cdot), \ldots, L_{6}(\cdot) \in C_{\text {per }}^{2}(Y)$ be the smooth functions introduced in Definition 5.1. We define the adapted function $\mathcal{A}_{\varepsilon}(f)(x, t)$ by

$$
\mathcal{A}_{\varepsilon}(f)(x, t):=f(x, t)+\sum_{i=1}^{6} \varepsilon^{i} L_{i}\left(\frac{x}{\varepsilon}\right) \partial_{x}^{i} f(x, t) .
$$

We now discuss the regularity of an adapted function $\mathcal{A}_{\varepsilon}(f)$.

Claim 3 Let $f(\cdot, t) \in H^{8}(\mathbb{R})$ for each $t$. Then

$$
\mathcal{A}_{\varepsilon}(f)(\cdot, t) \in H^{2}(\mathbb{R}) .
$$


Indeed, since $L_{i}(\cdot) \in C_{\text {per }}^{2}(Y)$, there exists an $\varepsilon$-independent constant $C$ such that

$$
\left\|\mathcal{A}_{\varepsilon}(f)(\cdot, t)\right\|_{H^{2}(\mathbb{R})}=\left\|f(\cdot, t)+\sum_{i=1}^{6} \varepsilon^{i} L_{i}\left(\frac{\dot{\varepsilon}}{\varepsilon}\right) \partial_{x}^{i} f(\cdot, t)\right\|_{H^{2}(\mathbb{R})} \leq \frac{C}{\varepsilon}\|f(\cdot, t)\|_{H^{8}(\mathbb{R})} .
$$

Our aim is to construct the auxiliary functions $L_{i}(y)$ such that (4.3) is satisfied. This is possible as we show in Appendix A. Actually, if the error $\rho=O\left(\varepsilon^{5}\right)$ and $m=4$, the functions $L_{i}(y)$ and the coefficients $a_{i}^{*}$ are uniquely determined, see Appendix A for details.

Next, let us make the implications of characterization (4.3) more precise. Setting $a^{\varepsilon}(x):=a\left(\frac{x}{\varepsilon}\right)$, one immediately discovers

Observation 2 Let $\mathcal{A}_{\varepsilon}$ be the adaption operator of Definition 4.2. Let $\mathcal{A}_{\varepsilon}$ satisfy characterization (4.3) with $m=4$ and let $f(x, t)$ solve the constant coefficient problem

$$
\varepsilon^{4} \partial_{t}^{2} f(x, t)-\sum_{i=0}^{4} \varepsilon^{i} a_{i}^{*} \partial_{x}^{2+i} f(x, t)=0
$$

Then the adaption of $f$ is an approximative solution of the time-scaled homogenization problem of Definition 2.1 in the sense that

$$
\begin{aligned}
& \varepsilon^{4} \partial_{t}^{2}\left(\mathcal{A}_{\varepsilon}(f)(x, t)\right)-\partial_{x}\left(a^{\varepsilon}(x) \partial_{x}\left(\mathcal{A}_{\varepsilon}(f)\right)(x, t)\right) \\
& =\mathcal{A}_{\varepsilon}\left(\varepsilon^{4} \partial_{t}^{2} f(x, t)-\sum_{i=0}^{4} \varepsilon^{i} a_{i}^{*} \partial_{x}^{2+i} f(x, t)\right)-\rho=\mathcal{A}_{\varepsilon}(0)-\rho=-\rho
\end{aligned}
$$

is small.

In the light of classical homogenization theory, we can expect for the first coefficient $a_{0}^{*}$ that $a_{0}^{*}=a^{*}=\left(c^{*}\right)^{2}$, where $c^{*}$ denotes the homogenized wave speed. This fact is also shown in Appendix A.

Finally, we make the connection between the adaption operator $\mathcal{A}_{\varepsilon}$ of Definition 4.2 and formula (4.3) more precise. Following the construction of Appendix A we insert $\mathcal{A}_{\varepsilon}(f)$ with appropriate $L_{i}(\cdot)$ in the left hand side of characterization (4.3).

Claim 3 suggests that the application of the differential operator $\partial_{x}\left(a^{\varepsilon}(x) \partial_{x}\right)$ to $\mathcal{A}_{\varepsilon}(f)(x, t)$ produces an $L^{2}(\mathbb{R})$-function.

Lemma 4.3 (Algebraical Lemma). Let $f(x, t) \in H^{8}(\mathbb{R})$ for each $t$ and let $\mathcal{A}_{\varepsilon}$ be the adaption operator of Definition 4.2. Then

$$
\begin{aligned}
\partial_{x}\left(a^{\varepsilon}(x) \partial_{x} \mathcal{A}_{\varepsilon}(f)(x, t)\right) \\
=a^{*} \partial_{x}^{2} f(x, t)+\varepsilon^{2} a_{2}^{*} \partial_{x}^{4} f(x, t)+\varepsilon^{4} a_{4}^{*} \partial_{x}^{6} f(x, t) \\
\quad+\varepsilon L_{1}\left(\frac{x}{\varepsilon}\right) \partial_{x}\left(a^{*} \partial_{x}^{2} f(x, t)+\varepsilon^{2} a_{2}^{*} \partial_{x}^{4} f(x, t)\right) \\
\quad+\varepsilon^{2} L_{2}\left(\frac{x}{\varepsilon}\right) \partial_{x}^{2}\left(a^{*} \partial_{x}^{2} f(x, t)+\varepsilon^{2} a_{2}^{*} \partial_{x}^{4} f(x, t)\right)
\end{aligned}
$$




$$
+\varepsilon^{3} L_{3}\left(\frac{x}{\varepsilon}\right) a^{*} \partial_{x}^{5} f(x, t)+\varepsilon^{4} L^{4}\left(\frac{x}{\varepsilon}\right) a^{*} \partial_{x}^{6} f(x, t)+\tilde{\rho}(x, t)
$$

with

$$
\begin{aligned}
\tilde{\rho}(x, t)= & \varepsilon^{5} \partial_{x}^{7} f(x, t) \partial_{y}\left(a(.) L_{6}(.)\right)\left(\frac{x}{\varepsilon}\right)+\varepsilon^{5} \partial_{x}^{7} f(x, t) a^{\varepsilon}(x)\left(L_{5}\left(\frac{x}{\varepsilon}\right)+\partial_{y} L_{6}\left(\frac{x}{\varepsilon}\right)\right) \\
& +\varepsilon^{6} a^{\varepsilon}(x) \partial_{x}^{8} f(x, t) L_{6}\left(\frac{x}{\varepsilon}\right) .
\end{aligned}
$$

Proof. The lemma follows from formula (5.2) and Lemmas 5.2 and 5.3 of the appendix.

Regarding the characterization (4.3), the Algebraical Lemma yields that

$$
\partial_{x}\left(a^{\varepsilon}(x) \partial_{x} \mathcal{A}_{\varepsilon}(f)(x, t)\right)=\mathcal{A}_{\varepsilon}\left(a^{*} \partial_{x}^{2} f(x, t)+\varepsilon^{2} a_{2}^{*} \partial_{x}^{4} f(x, t)+\varepsilon^{4} a_{4}^{*} \partial_{x}^{6} f(x, t)\right)+O\left(\varepsilon^{5}\right)
$$

in $L^{2}(\mathbb{R})$ independent of $t$. We consider only terms up to the fourth derivative of $f$. Observation 2, exploiting $a_{1}^{*}=0, a_{3}^{*}=0$, then suggests to consider the problem

$$
\varepsilon^{4} \partial_{t}^{2} f(x, t)-a^{*} \partial_{x}^{2} f(x, t)-\varepsilon^{2} a_{2}^{*} \partial_{x}^{4} f(x, t)=0 .
$$

Remark: Keeping in mind that $a_{2}^{*}>0$, we observe that Problem (4.5) is ill-posed. However, to lowest order, one formally can replace $\partial_{x}^{4} f$ by $\frac{\varepsilon^{4}}{a^{*}} \partial_{t}^{2} \partial_{x}^{2} f$ arriving at the weakly dispersive equation of Definition 2.2.

\subsection{Proof of Theorem 1}

We are now in the position to prove Theorem 1. In what follows, $u_{\varepsilon}$ denotes the solution of the time-scaled homogenization problem of Definition 2.1 and $v_{\varepsilon}$ the weakly dispersive solution of Definition 2.2. Actually, in our first step we show that the difference between $u_{\varepsilon}$ and $\mathcal{A}_{\varepsilon}\left(v_{\varepsilon}\right)$ is small. In the second step the adaption $\mathcal{A}_{\varepsilon}$ is omitted. Our first result is analogous to Proposition 3.5.

Proof of Theorem 1.

First step. The crucial point is to show that there exists an $\varepsilon$-independent constant $C$ such that

$$
\begin{aligned}
\left\|\partial_{x} u_{\varepsilon}(x, t)-\partial_{x}\left(\mathcal{A}_{\varepsilon}\left(v_{\varepsilon}\right)\right)(x, t)\right\|_{L^{\infty}\left(0, T ; L^{2}(\mathbb{R})\right)} & \leq C \varepsilon^{2} \\
\left\|u_{\varepsilon}(x, t)-\left(\mathcal{A}_{\varepsilon}\left(v_{\varepsilon}\right)\right)(x, t)\right\|_{L^{\infty}\left(0, T ; L^{2}(\mathbb{R})\right)} & \leq C .
\end{aligned}
$$

The idea is to apply the partial differential operator $\varepsilon^{4} \partial_{t}^{2}-\partial_{x}\left(a^{\varepsilon}(x) \partial_{x}\right)$ to $\mathcal{A}_{\varepsilon}\left(v_{\varepsilon}\right)$. The difference between $u_{\varepsilon}$ and $\mathcal{A}_{\varepsilon}\left(v_{\varepsilon}\right)$ is then controlled by standard energy estimates.

In the subsequent calculation, equality (1) holds due to the Algebraical Lemma 4.3. Using in (2) that $v_{\varepsilon}$ solves the weakly dispersive problem of Definition 2.2 $\varepsilon^{4} \partial_{t}^{2} v_{\varepsilon}-a^{*} \partial_{x}^{2} v_{\varepsilon}=\varepsilon^{6} \frac{a_{2}^{*}}{a^{*}} \partial_{t}^{2} \partial_{x}^{2} v_{\varepsilon}$, we obtain

$$
\begin{aligned}
& \varepsilon^{4} \partial_{t}^{2}\left(\mathcal{A}_{\varepsilon} v_{\varepsilon}\right)(x, t)-\partial_{x}\left(a^{\varepsilon}(x) \partial_{x}\left(\mathcal{A}_{\varepsilon} v_{\varepsilon}\right)(x, t)\right) \\
& \quad \stackrel{(1)}{=} \varepsilon^{4} \partial_{t}^{2} v_{\varepsilon}(x, t)-a^{*} \partial_{x}^{2} v_{\varepsilon}(x, t)-\varepsilon^{2} a_{2}^{*} \partial_{x}^{4} v_{\varepsilon}(x, t)-\varepsilon^{4} a_{4}^{*} \partial_{x}^{6} v_{\varepsilon}(x, t)
\end{aligned}
$$




$$
\begin{aligned}
+ & \varepsilon L_{1}\left(\frac{x}{\varepsilon}\right) \partial_{x}\left(\varepsilon^{4} \partial_{t}^{2} v_{\varepsilon}(x, t)-a^{*} \partial_{x}^{2} v_{\varepsilon}(x, t)-\varepsilon^{2} a_{2}^{*} \partial_{x}^{4} v_{\varepsilon}(x, t)\right) \\
& +\varepsilon^{2} L_{2}\left(\frac{x}{\varepsilon}\right) \partial_{x}^{2}\left(\varepsilon^{4} \partial_{t}^{2} v_{\varepsilon}(x, t)-a^{*} \partial_{x}^{2} v_{\varepsilon}(x, t)-\varepsilon^{2} a_{2}^{*} \partial_{x}^{4} v_{\varepsilon}(x, t)\right) \\
& +\varepsilon^{3} L_{3}\left(\frac{x}{\varepsilon}\right) \partial_{x}^{3}\left(\varepsilon^{4} \partial_{t}^{2} v_{\varepsilon}(x, t)-a^{*} \partial_{x}^{2} v_{\varepsilon}(x, t)\right) \\
& +\varepsilon^{4} L_{4}\left(\frac{x}{\varepsilon}\right) \partial_{x}^{4}\left(\varepsilon^{4} \partial_{t}^{2} v_{\varepsilon}(x, t)-a^{*} \partial_{x}^{2} v_{\varepsilon}(x, t)\right) \\
& +\varepsilon^{9} L_{5}\left(\frac{x}{\varepsilon}\right) \partial_{x}^{5} \partial_{t}^{2} v_{\varepsilon}(x, t)+\varepsilon^{10} L_{6}\left(\frac{x}{\varepsilon}\right) \partial_{x}^{6} \partial_{t}^{2} v_{\varepsilon}(x, t) \\
& -\varepsilon^{5} \partial_{x}^{7} v_{\varepsilon}(x, t)\left(\partial_{y}\left(a(.) L_{6}(.)\right)\left(\frac{x}{\varepsilon}\right)+a^{\varepsilon}(x)\left(L_{5}\left(\frac{x}{\varepsilon}\right)-\partial_{y} L_{6}\left(\frac{x}{\varepsilon}\right)\right)\right) \\
& -\varepsilon^{6} a^{\varepsilon}(x) \partial_{x}^{8} v_{\varepsilon}(x, t) L_{6}\left(\frac{x}{\varepsilon}\right) \\
\frac{(2)}{=} & a_{2}^{*} \varepsilon^{2}\left(\frac{\varepsilon^{4}}{a^{*}} \partial_{t}^{2} \partial_{x}^{2} v_{\varepsilon}-\partial_{x}^{4} v_{\varepsilon}\right)(x, t)+a_{2}^{*} \varepsilon^{3} L_{1}\left(\frac{x}{\varepsilon}\right)\left(\frac{\varepsilon^{4}}{a^{*}} \partial_{t}^{2} \partial_{x}^{3} v_{\varepsilon}-\partial_{x}^{5} v_{\varepsilon}\right)(x, t) \\
& +a_{2}^{*} \varepsilon^{4} L_{2}\left(\frac{x}{\varepsilon}\right)\left(\frac{\varepsilon^{4}}{a^{*}} \partial_{t}^{2} \partial_{x}^{4} v_{\varepsilon}-\partial_{x}^{6} v_{\varepsilon}\right)(x, t) \\
& -\varepsilon^{4} a_{4}^{*} \partial_{x}^{6} v_{\varepsilon}(x, t)-\varepsilon^{5} \partial_{x}^{7} v_{\varepsilon}(x, t)\left(\partial_{y}\left(a(.) L_{6}(.)\right)\left(\frac{x}{\varepsilon}\right) a^{\varepsilon}(x)\left(L_{5}(.)+\partial_{y} L_{6}(.)\right)\left(\frac{x}{\varepsilon}\right)\right) \\
& -\varepsilon^{6} a^{\varepsilon}(x) L_{6}\left(\frac{x}{\varepsilon}\right) \partial_{x}^{8} v_{\varepsilon}(x, t)+\varepsilon^{9} \partial_{t}^{2} \partial_{x}^{5} v_{\varepsilon}(x, t)\left(\frac{a_{2}^{*}}{a^{*}} L_{3}\left(\frac{x}{\varepsilon}\right)+L_{5}\left(\frac{x}{\varepsilon}\right)\right) \\
& +\varepsilon^{10} \partial_{t}^{2} \partial_{x}^{6} v_{\varepsilon}(x, t)\left(\frac{a_{2}^{*}}{a^{*}} L_{4}\left(\frac{x}{\varepsilon}\right)+L_{6}\left(\frac{x}{\varepsilon}\right)\right) .
\end{aligned}
$$

To sum up, the effect of the wave operator on $\mathcal{A}_{\varepsilon}\left(v_{\varepsilon}\right)$ is characterized by

$$
\varepsilon^{4} \partial_{t}^{2}\left(\mathcal{A}_{\varepsilon} v_{\varepsilon}\right)(x, t)-\partial_{x}\left(a^{\varepsilon}(x) \partial_{x}\left(\mathcal{A}_{\varepsilon} v_{\varepsilon}\right)(x, t)\right)=f_{\varepsilon}(x, t)
$$

The estimates for $f_{\varepsilon}$ are obtained in a quite elementary way and can be found in Appendix B. They provide

$$
\left\|f_{\varepsilon}(x, t)\right\|_{L^{2}\left(0, T ; L^{2}(\mathbb{R})\right)} \leq C \varepsilon^{4} .
$$

Now, we consider the energy estimate for the time-scaled homogenization problem of Definition 2.1 and apply it to $u_{\varepsilon}-\mathcal{A}_{\varepsilon}\left(v_{\varepsilon}\right)$. We refer to Appendix $\mathrm{C}$ for details. Let us remark that due to classical regularity theory, Proposition 4.1 and Claim $3, u_{\varepsilon}$ and $\mathcal{A}_{\varepsilon}\left(v_{\varepsilon}\right)$ are sufficiently regular.

Exploiting estimates for the initial data of Appendix B,

$$
\begin{gathered}
\left\|\partial_{x} u_{\varepsilon}(x, 0)-\partial_{x}\left(\mathcal{A}_{\varepsilon}\left(v_{\varepsilon}\right)\right)(x, 0)\right\|_{L^{2}(\mathbb{R})}^{2} \leq C \varepsilon^{4} \\
\varepsilon^{4}\left\|\partial_{t} u_{\varepsilon}(x, 0)-\partial_{t}\left(\mathcal{A}_{\varepsilon}\left(v_{\varepsilon}\right)\right)(x, 0)\right\|_{L^{2}(\mathbb{R})}^{2} \leq C \varepsilon^{4}
\end{gathered}
$$

and taking into account that $u_{\varepsilon}$ solves the time-scaled homogenization problem, one arrives at

$$
\left\|\partial_{x} u_{\varepsilon}-\partial_{x}\left(\mathcal{A}_{\varepsilon}\left(v_{\varepsilon}\right)\right)\right\|_{L^{\infty}\left(0, T ; L^{2}(\mathbb{R})\right)}^{2}+\varepsilon^{4}\left\|\partial_{t}\left(u_{\varepsilon}\right)-\partial_{t}\left(\mathcal{A}_{\varepsilon}\left(v_{\varepsilon}\right)\right)\right\|_{L^{\infty}\left(0, T ; L^{2}(\mathbb{R})\right)}^{2}
$$




$$
\begin{aligned}
\leq & C\left(\left\|\partial_{x} u_{\varepsilon}(x, 0)-\partial_{x}\left(\mathcal{A}_{\varepsilon}\left(v_{\varepsilon}\right)\right)(x, 0)\right\|_{L^{2}(\mathbb{R})}^{2}\right. \\
& \left.+\varepsilon^{4}\left\|\partial_{t} u_{\varepsilon}(x, 0)-\partial_{t}\left(\mathcal{A}_{\varepsilon}\left(v_{\varepsilon}\right)\right)(x, 0)\right\|_{L^{2}(\mathbb{R})}^{2}+\frac{1}{\varepsilon^{4}}\left\|f_{\varepsilon}\right\|_{L^{2}\left(0, T ; L^{2}(\mathbb{R})\right)}^{2}\right) \\
\leq & C \varepsilon^{4} .
\end{aligned}
$$

Now (4.6) is a direct consequence of (4.9). Moreover, one obtains

$$
\left\|\partial_{t} u_{\varepsilon}-\partial_{t} \mathcal{A}_{\varepsilon}\left(v_{\varepsilon}\right)\right\|_{L^{\infty}\left(0, T ; L^{2}(\mathbb{R})\right)} \leq C
$$

and thus, writing

$$
\left(u_{\varepsilon}-\mathcal{A}_{\varepsilon}\left(v_{\varepsilon}\right)\right)(x, t)=\left(u_{\varepsilon}-\mathcal{A}_{\varepsilon}\left(v_{\varepsilon}\right)\right)(x, 0)+\int_{0}^{t} \partial_{t}\left(u_{\varepsilon}-\mathcal{A}_{\varepsilon}\left(v_{\varepsilon}\right)\right)(x, \tau) d \tau,
$$

inequality (4.7) follows directly.

Second step. We would like to show that

$$
\left\|u_{\varepsilon}(x, t)-v_{\varepsilon}(x, t)\right\|_{L^{\infty}\left(0, T ; L^{\infty}(\mathbb{R})\right)} \stackrel{\varepsilon \rightarrow 0}{\rightarrow} 0 .
$$

Arguing in exactly the same way as in the proof of Theorem 2, inequalities (4.6), (4.7) yield

$$
\left\|u_{\varepsilon}(x, t)-\mathcal{A}_{\varepsilon}\left(v_{\varepsilon}\right)(x, t)\right\|_{L^{\infty}\left(0, T ; L^{\infty}(\mathbb{R})\right)} \stackrel{\varepsilon \rightarrow 0}{\rightarrow} 0 .
$$

It remains to avoid the adaption operator $\mathcal{A}_{\varepsilon}$. Actually, in the subsequent calculation we use in $(1)$ that $\left\|L_{1}\left(\frac{x}{\varepsilon}\right)\right\|_{L^{\infty}(\mathbb{R})}, \ldots,\left\|L_{6}\left(\frac{x}{\varepsilon}\right)\right\|_{L^{\infty}(\mathbb{R})}$ are uniformly bounded in $\varepsilon$. Inequality (2) is a consequence of Proposition 4.1 using once more the fundamental theorem of calculus.

$$
\begin{aligned}
& \left\|\mathcal{A}_{\varepsilon}\left(v_{\varepsilon}\right)(x, t)-v_{\varepsilon}(x, t)\right\|_{L^{\infty}\left(0, T ; L^{\infty}(\mathbb{R})\right)} \\
= & \left\|\varepsilon L_{1}\left(\frac{x}{\varepsilon}\right) \partial_{x} v_{\varepsilon}(x, t)+\ldots+\varepsilon^{6} L_{6}\left(\frac{x}{\varepsilon}\right) \partial_{x}^{6} v_{\varepsilon}(x, t)\right\|_{L^{\infty}\left(0, T ; L^{\infty}(\mathbb{R})\right)} \\
\stackrel{(1)}{\leq} & C \varepsilon\left(\left\|\partial_{x} v_{\varepsilon}(x, t)\right\|_{L^{\infty}\left(0, T ; L^{\infty}(\mathbb{R})\right)}+\ldots+\left\|\partial_{x}^{6} v_{\varepsilon}(x, t)\right\|_{L^{\infty}\left(0, T ; L^{\infty}(\mathbb{R})\right)}\right) \\
\stackrel{(2)}{\leq} & C \varepsilon .
\end{aligned}
$$

This proves Theorem 1 .

\section{Appendix}

Appendix A: Construction of $\mathcal{A}_{\varepsilon}$ and main properties. According to characterization (4.3), we derive in Appendix A.1 the periodic boundary problems which determine the auxiliary functions $L_{1}(y), \ldots, L_{6}(y)$. Algebraical simplifications are performed in Appendix A.2. The Algebraical Lemma 4.3 is a direct consequence of the results in Appendix A.1 and A.2. 
Appendix A.1: Construction of the auxiliary problems. Our aim is to construct the adaption operator $\mathcal{A}_{\varepsilon}$ such that

$$
\begin{aligned}
& \partial_{x}\left(a^{\varepsilon}(x) \partial_{x} \mathcal{A}_{\varepsilon}(f)(x, t)\right) \\
& =\mathcal{A}_{\varepsilon}\left(\sum_{i=0}^{4} \varepsilon^{i} a_{i}^{*} \partial_{x}^{i+2} f(x, t)\right)+O\left(\varepsilon^{5}\right)
\end{aligned}
$$

for every smooth function $f$. We calculate the left hand side of (5.1) as

$$
\begin{aligned}
& \left.\partial_{x}\left(a^{\varepsilon}(x) \partial_{x}\left(\mathcal{A}_{\varepsilon}(f)\right)(x, t)\right)\right) \\
= & \partial_{x}\left(a^{\varepsilon}(x)\left(1+\partial_{y} L_{1}\left(\frac{x}{\varepsilon}\right)\right) \partial_{x} f(x, t)+\sum_{i=2}^{6} \varepsilon^{i-1} a^{\varepsilon}(x)\left(L_{i-1}(.)+\partial_{y} L_{i}(.)\right)\left(\frac{x}{\varepsilon}\right) \partial_{x}^{i} f(x, t)\right) \\
& +\partial_{x}\left(\varepsilon^{6} a^{\varepsilon}(x) L_{6}\left(\frac{x}{\varepsilon}\right) \partial_{x}^{7} f(x, t)\right) \\
= & \frac{1}{\varepsilon} \partial_{x} f(x, t) \partial_{y}\left(a(.)\left(1+\partial_{y} L_{1}(.)\right)\right)\left(\frac{x}{\varepsilon}\right)+\partial_{x}^{2} f(x, t) a^{\varepsilon}(x)\left(1+\partial_{y} L_{1}\left(\frac{x}{\varepsilon}\right)\right) \\
& +\sum_{i=1}^{5} \varepsilon^{i-1} \partial_{y}\left(a(.)\left(L_{i}(.)+\partial_{y} L_{i+1}(.)\right)\right)\left(\frac{x}{\varepsilon}\right) \partial_{x}^{i+1} f(x, t) \\
& +\sum_{i=2}^{6} \varepsilon^{i-1} a^{\varepsilon}(x)\left(L_{i-1}(.)+\partial_{y} L_{i}(.)\right)\left(\frac{x}{\varepsilon}\right) \partial_{x}^{i+1} f(x, t) \\
& +\varepsilon^{5} \partial_{y}\left(a(.) L_{6}(.)\right)\left(\frac{x}{\varepsilon}\right) \partial_{x}^{7} f(x, t)+\varepsilon^{6} a^{\varepsilon}(x) L_{6}\left(\frac{x}{\varepsilon}\right) \partial_{x}^{8} f(x, t) .
\end{aligned}
$$

Considering terms of order $(1 / \varepsilon)$ in $(5.1)$, one immediately derives the following equation for $L_{1}(y)$

$$
\partial_{y}\left(a(y)\left(1+\partial_{y} L_{1}(y)\right)\right)=0 .
$$

The additional claim $\left\langle L_{1}(y)\right\rangle_{Y}=0$ makes the periodic boundary problem (5.3) wellposed. Hence, a unique solution $L_{1}(y) \in H_{\text {per }}^{1}(Y)$ exists, see [5] for details.

At order (1), as a pre-factor of $\partial_{x}^{2} f$, the following equation arises

$$
\partial_{y}\left(a(y)\left(L_{1}(y)+\partial_{y} L_{2}(y)\right)\right)=a_{0}^{*}-a(y)\left(1+\partial_{y} L_{1}(y)\right) .
$$

Considering the mean value

$$
\begin{aligned}
0=\left\langle\partial_{y}\left(a(y)\left(L_{1}(y)+\partial_{y} L_{2}(y)\right)\right)\right\rangle_{Y} & =\left\langle a_{0}^{*}-a(y)\left(1+\partial_{y} L_{1}(y)\right)\right\rangle_{Y} \\
& =a_{0}^{*}-\left\langle a(y)\left(1+\partial_{y} L_{1}(y)\right)\right\rangle_{Y}
\end{aligned}
$$

one directly concludes $a_{0}^{*}=\left\langle a(y)\left(1+\partial_{y} L_{1}(y)\right)\right\rangle_{Y}$. Demanding $\left\langle L_{2}(y)\right\rangle_{Y}=0$, problem (5.4) determines $L_{2}(y)$ uniquely.

Next, considering terms of order $(\varepsilon)$, one finds as pre-factor of $\partial_{x}^{3} f$

$$
\partial_{y}\left(a(y)\left(L_{2}(y)+\partial_{y} L_{3}(y)\right)\right)=a_{1}^{*}+a_{0}^{*} L_{1}(y)-a(y)\left(L_{1}(y)+\partial_{y} L_{2}(y)\right),
$$


where $a_{1}^{*}=\left\langle a(y)\left(L_{1}(y)+\partial_{y} L_{2}(y)\right)\right\rangle_{Y}$, since

$$
\begin{aligned}
0=\left\langle\partial_{y}\left(a(y)\left(L_{2}(y)+\partial_{y} L_{3}(y)\right)\right)\right\rangle_{Y} & =\left\langle a_{1}^{*}+a_{0}^{*} L_{1}(y)-a(y)\left(L_{1}(y)+\partial_{y} L_{2}(y)\right)\right\rangle_{Y} \\
& =a_{1}^{*}-\left\langle a(y)\left(L_{1}(y)+\partial_{y} L_{2}(y)\right)\right\rangle_{Y} .
\end{aligned}
$$

Then a unique solution to problem (5.5), with $\left\langle L_{3}(y)\right\rangle_{Y}=0$, exists. In an analogous manner, the equation at order $\left(\varepsilon^{2}\right)$ is given by

$$
\begin{aligned}
\partial_{y}(a(y) & \left.\left(L_{3}(y)+\partial_{y} L_{4}(y)\right)\right) \\
= & a_{2}^{*}+a_{1}^{*} L_{1}(y)+a_{0}^{*} L_{2}(y)-a(y)\left(L_{2}(y)+\partial_{y} L_{3}(y)\right) .
\end{aligned}
$$

Again, the mean value yields

$$
\begin{aligned}
0 & =\left\langle\partial_{y}\left(a(y)\left(L_{2}(y)+\partial_{y} L_{3}(y)\right)\right)\right\rangle_{Y} \\
& =\left\langle a_{2}^{*}+a_{1}^{*} L_{1}(y)+a_{0}^{*} L_{2}(y)-a(y)\left(L_{2}(y)+\partial_{y} L_{3}(y)\right)\right\rangle_{Y} \\
& =a_{2}^{*}-\left\langle a(y)\left(L_{2}(y)+\partial_{y} L_{3}(y)\right)\right\rangle_{Y}
\end{aligned}
$$

and thus $a_{2}^{*}=\left\langle a(y)\left(L_{2}(y)+\partial_{y} L_{3}(y)\right)\right\rangle_{Y}$. Problem (5.6) uniquely determines $L_{4}(y)$ with $\left\langle L_{4}(y)\right\rangle_{Y}=0$. Following this procedure up to order $\left(\varepsilon^{4}\right)$, one at least arrives at the following definition. By smoothness of $a(\cdot)$, the auxiliary functions are smooth, $L_{1}(y), \ldots, L_{6}(y) \in C_{p e r}^{2}(Y)$.

Definition 5.1 (Auxiliary problems). Denote by $L_{1}(y), \ldots, L_{6}(y)$ the unique solution to the following problem

$$
\begin{aligned}
& \partial_{y}\left(a(y)\left(1+\partial_{y} L_{1}(y)\right)\right)=0 \\
& \partial_{y}\left(a(y)\left(L_{1}(y)+\partial_{y} L_{2}(y)\right)\right)= a_{0}^{*}-a(y)\left(1+\partial_{y} L_{1}(y)\right), \\
& \partial_{y}\left(a(y)\left(L_{2}(y)+\partial_{y} L_{3}(y)\right)\right)= a_{1}^{*}+a_{0}^{*} L_{1}(y)-a(y)\left(L_{1}(y)+\partial_{y} L_{2}(y)\right), \\
& \partial_{y}\left(a(y)\left(L_{3}(y)+\partial_{y} L_{4}(y)\right)\right)= a_{2}^{*}+a_{1}^{*} L_{1}(y)+a_{0}^{*} L_{2}(y)-a(y)\left(L_{2}(y)+\partial_{y} L_{3}(y)\right), \\
& \partial_{y}\left(a(y)\left(L_{4}(y)+\partial_{y} L_{5}(y)\right)\right)= a_{3}^{*}+a_{2}^{*} L_{1}(y)+a_{1}^{*} L_{2}(y)+a_{0}^{*} L_{3}(y) \\
&-a(y)\left(L_{3}(y)+\partial_{y} L_{4}(y)\right), \\
& \partial_{y}\left(a(y)\left(L_{5}(y)+\partial_{y} L_{6}(y)\right)\right)= a_{4}^{*}+a_{3}^{*} L_{1}(y)+a_{2}^{*} L_{2}(y)+a_{1}^{*} L_{3}(y)+a_{0}^{*} L_{4}(y) \\
&-a(y)\left(L_{4}(y)+\partial_{y} L_{5}(y)\right)
\end{aligned}
$$

and

$$
\left\langle L_{1}(y)\right\rangle_{Y}=\left\langle L_{2}(y)\right\rangle_{Y}=\left\langle L_{3}(y)\right\rangle_{Y}=\left\langle L_{4}(y)\right\rangle_{Y}=\left\langle L_{5}(y)\right\rangle_{Y}=\left\langle L_{6}(y)\right\rangle_{Y}=0
$$

where

$$
\begin{aligned}
& a_{0}^{*}:=\left\langle a(y)\left(1+\partial_{y} L_{1}(y)\right)\right\rangle_{Y}, \\
& a_{i}^{*}:=\left\langle a(y)\left(L_{i}(y)+\partial_{y} L_{i+1}(y)\right)\right\rangle_{Y},
\end{aligned}
$$

for $1 \leq i \leq 4$. 


\section{Appendix A.2 Algebraical simplifications.}

Lemma 5.2. The auxiliary functions $L_{1}(y), L_{2}(y) \in C_{p e r}^{2}(Y)$ satisfy

$$
\begin{aligned}
a(y)\left(1+\partial_{y} L_{1}(y)\right) & =a_{0}^{*}=a^{*}, \\
a(y)\left(L_{1}(y)+\partial_{y} L_{2}(y)\right) & =a_{1}^{*}=0 .
\end{aligned}
$$

Proof. Since $a_{0}^{*}=\left\langle a(y)\left(1+\partial_{y} L_{1}(y)\right)\right\rangle_{Y}$, one obtains

$$
\begin{aligned}
\partial_{y}\left(a(y)\left(1+\partial_{y} L_{1}(y)\right)\right)=0 \quad \Rightarrow \quad a(y)\left(1+\partial_{y} L_{1}(y)\right)=a_{0}^{*} \\
\Rightarrow 1+\partial_{y} L_{1}(y)=\frac{a_{0}^{*}}{a(y)} \Rightarrow\left\langle 1+\partial_{y} L_{1}(y)\right\rangle_{Y}=a_{0}^{*}\left\langle\frac{1}{a(y)}\right\rangle_{Y} .
\end{aligned}
$$

Due to $L_{1}(y) \in C_{p e r}^{2}(Y)$ and thus $\left\langle\partial_{y} L_{1}(y)\right\rangle_{Y}=0$, we conclude

$$
1=a_{0}^{*}\left\langle\frac{1}{a(y)}\right\rangle_{Y}=\frac{a_{0}^{*}}{a^{*}} \quad \Rightarrow \quad a_{0}^{*}=a^{*} .
$$

Inserting in the equation for $L_{2}$, it follows that $a(y)\left(L_{1}(y)+\partial_{y} L_{2}(y)\right)=0$. This provides $a_{1}^{*}=0$ in the next equation.

Lemma 5.3 (The mean value $a_{3}^{*}$ ). Consider the mean value $a_{3}^{*}$ of Definition 5.1. Then in fact

$$
a_{3}^{*}=0
$$

Proof. In what follows, equalities (1) and (3) are direct consequences of Definition 5.1 and Lemma 5.2. Equality (2) is valid, since $a(y)\left(L_{1}(y)+\partial_{y} L_{2}(y)\right)=0$ and thus $\partial_{y} L_{2}(y)=-L_{1}(y)$. We calculate

$$
\begin{aligned}
& \partial_{y}\left(\partial_{y}\left(a(y)\left(L_{3}(y)+\partial_{y} L_{4}(y)\right)\right)\right) \\
& =\partial_{y}\left(a_{2}^{*}+a^{*} L_{2}(y)-a(y)\left(L_{2}(y)+\partial_{y} L_{3}(y)\right)\right) \stackrel{(1)}{=} a^{*} \partial_{y} L_{2}(y)-a^{*} L_{1}(y) \\
& \stackrel{(2)}{=}-2 a^{*} L_{1}(y) \stackrel{(3)}{=}-2 \partial_{y}\left(a(y)\left(L_{2}(y)+\partial_{y} L_{3}(y)\right)\right) .
\end{aligned}
$$

Consequently,

$$
\partial_{y}\left(a(y)\left(L_{3}(y)+\partial_{y} L_{4}(y)\right)\right)=-2 a(y)\left(L_{2}(y)+\partial_{y} L_{3}(y)\right)+C
$$

for a constant $C$. Considering the mean value one discovers

$$
0=-2 a_{2}^{*}+C
$$

and thus

$$
\begin{aligned}
\partial_{y}\left(a(y)\left(L_{3}(y)+\partial_{y} L_{4}(y)\right)\right) & =-2 a(y)\left(L_{2}(y)+\partial_{y} L_{3}(y)\right)+2 a_{2}^{*} \\
& =a_{2}^{*}+a^{*} L_{2}(y)-a(y)\left(L_{2}(y)+\partial_{y} L_{3}(y)\right) .
\end{aligned}
$$


The last equality is valid due to Definition 5.1. Consequently,

$$
-a(y)\left(L_{2}(y)+\partial_{y} L_{3}(y)\right)+a_{2}^{*}=a^{*} L_{2}(y)
$$

and thus

$$
\partial_{y}\left(a(y)\left(L_{3}(y)+\partial_{y} L_{4}(y)\right)\right)=2 a^{*} L_{2}(y) .
$$

Next, multiplying equation (5.9) by $L_{1}(y)$, integrating over Y and applying integration by parts yields

$$
\begin{aligned}
-\int_{Y} \partial_{y} L_{1}(y) a(y)\left(L_{3}(y)+\partial_{y} L_{4}(y)\right) d y & =2 a^{*} \int_{Y} L_{2}(y) L_{1}(y) d y \\
\stackrel{(1)}{=}-2 a^{*} \int_{Y} L_{2}(y) \partial_{y} L_{2}(y) d y & =-a^{*} \int_{Y} \partial_{y}\left(L_{2}(y)\right)^{2} d y=0,
\end{aligned}
$$

where (1) again uses the fact that $\partial_{y} L_{2}(y)=-L_{1}(y)$. Moreover, taking into account $\partial_{y} L_{1}(y)=\frac{a^{*}}{a(y)}-1$, see Lemma 5.2 , one concludes

$$
0=\int_{Y} a(y)\left(L_{3}+\partial_{y} L_{4}\right)(y) d y-\int_{Y} a^{*}\left(L_{3}+\partial_{y} L_{4}\right)(y) d y=\int_{Y} a(y)\left(L_{3}+\partial_{y} L_{4}\right)(y) d y
$$

which is the claimed result.

Remark 5.4 (The mean value $a_{2}^{*}$ ). Consider the mean value $a_{2}^{*}$ of Definition 5.1. Then

$$
a_{2}^{*}>0
$$

Proof. As in the proof of Lemma 5.3, one multiplies the equation

$$
\partial_{y}\left(a(y)\left(L_{2}(y)+\partial_{y} L_{3}(y)\right)\right)=a^{*} L_{1}(y)
$$

by $L_{1}(y)$, integrates over $Y$ and applies integration by parts arriving at

$$
-\int_{Y} \partial_{y} L_{1}(y)\left(a(y)\left(L_{2}(y)+\partial_{y} L_{3}(y)\right)\right) d y=a^{*}\left\langle L_{1}(y) L_{1}(y)\right\rangle_{Y}>0 .
$$

Now, we again use that $\partial_{y} L_{1}(y)=\frac{a^{*}}{a(y)}-1$. Consequently,

$$
\begin{aligned}
-\int_{Y} \partial_{y} & L_{1}(y)\left(a(y)\left(L_{2}(y)+\partial_{y} L_{3}(y)\right)\right) d y \\
& =\int_{Y}\left(a(y)\left(L_{2}(y)+\partial_{y} L_{3}(y)\right) d y-\int_{Y} a^{*}\left(L_{2}(y)+\partial_{y} L_{3}(y)\right) d y\right. \\
& =\int_{Y}\left(a(y)\left(L_{2}(y)+\partial_{y} L_{3}(y)\right) d y=a_{2}^{*}\right.
\end{aligned}
$$

and thus the positivity of $a_{2}^{*}$.

The Algebraical Lemma 4.3 directly follows considering Definition 5.1 and inserting in (5.2) the results of Lemma 5.2 and Lemma 5.3. 
Appendix B. In this appendix we show some estimates which are applied in the proof of Theorem 1 .

Appendix B1: Effect of the wave operator on $\mathcal{A}_{\varepsilon}\left(v_{\varepsilon}\right)$. Our aim is to estimate the \|\|$_{L^{2}\left(0, T ; L^{2}(\mathbb{R})\right)^{-n o r m} \text { of the following expressions }}$

$$
\begin{aligned}
& \text { a) } a_{2}^{*} \varepsilon^{2}\left(\frac{\varepsilon^{4}}{a^{*}} \partial_{t}^{2} \partial_{x}^{2} v_{\varepsilon}-\partial_{x}^{4} v_{\varepsilon}\right)(x, t), \\
& \text { b) } a_{2}^{*} \varepsilon^{3} L_{1}\left(\frac{x}{\varepsilon}\right)\left(\frac{\varepsilon^{4}}{a^{*}} \partial_{t}^{2} \partial_{x}^{3} v_{\varepsilon}-\partial_{x}^{5} v_{\varepsilon}\right)(x, t), \\
& \text { c) } a_{2}^{*} \varepsilon^{4} L_{2}\left(\frac{x}{\varepsilon}\right)\left(\frac{\varepsilon^{4}}{a^{*}} \partial_{t}^{2} \partial_{x}^{4} v_{\varepsilon}-\partial_{x}^{6} v_{\varepsilon}\right)(x, t), \\
& \text { d) } \varepsilon^{4} a_{4}^{*} \partial_{x}^{6} v_{\varepsilon}(x, t) \\
& \text { e) } \varepsilon^{5} \partial_{x}^{7} v_{\varepsilon}(x, t)\left(a^{\varepsilon}(x)\left(L_{5}(.)+\partial_{y} L_{6}(.)\right)\left(\frac{x}{\varepsilon}\right)+\partial_{y}\left(a(.) L_{6}(.)\right)\left(\frac{x}{\varepsilon}\right)\right), \\
& \text { f) } \varepsilon^{6} L_{6}\left(\frac{x}{\varepsilon}\right) a^{\varepsilon}(x) \partial_{x}^{8} v_{\varepsilon}(x, t), \\
& \text { g) } \varepsilon^{9}\left(\frac{a_{2}^{*}}{a^{*}} L_{3}\left(\frac{x}{\varepsilon}\right)+L_{5}\left(\frac{x}{\varepsilon}\right)\right) \partial_{t}^{2} \partial_{x}^{5} v_{\varepsilon}(x, t), \\
& \text { h) } \varepsilon^{10}\left(\frac{a_{2}^{*}}{a^{*}} L_{4}\left(\frac{x}{\varepsilon}\right)+L_{6}\left(\frac{x}{\varepsilon}\right)\right) \partial_{t}^{2} \partial_{x}^{6} v_{\varepsilon}(x, t) .
\end{aligned}
$$

In what follows, we exploit that due to $L_{i}(y) \in C_{\text {per }}^{2}(Y)$, there exists an $\varepsilon$ - independent constant $K>0$, such that

$$
\begin{aligned}
\left\|L_{1}\left(\frac{x}{\varepsilon}\right)\right\|_{L^{\infty}(\mathbb{R})}, \ldots,\left\|L_{6}\left(\frac{x}{\varepsilon}\right)\right\|_{L^{\infty}(\mathbb{R})} & \leq K, \\
\left\|\partial_{y} L_{1}\left(\frac{x}{\varepsilon}\right)\right\|_{L^{\infty}(\mathbb{R})}, \ldots,\left\|\partial_{y} L_{6}\left(\frac{x}{\varepsilon}\right)\right\|_{L^{\infty}(\mathbb{R})} & \leq K .
\end{aligned}
$$

a) We start with expression a). Due to

$$
\begin{aligned}
\left(\frac{\varepsilon^{4}}{a^{*}} \partial_{t}^{2} \partial_{x}^{2} v_{\varepsilon}-\partial_{x}^{4} v_{\varepsilon}\right)(x, t) & =\partial_{x}^{2}\left(\frac{\varepsilon^{4}}{a^{*}} \partial_{t}^{2} v_{\varepsilon}-\partial_{x}^{2} v_{\varepsilon}\right)(x, t)=\frac{\varepsilon^{6} a_{2}^{*}}{\left(a^{*}\right)^{2}} \partial_{t}^{2} \partial_{x}^{4} v_{\varepsilon}(x, t) \\
& =\frac{\varepsilon^{2} a_{2}^{*}}{\left(a^{*}\right)^{2}} \partial_{x}^{4}\left(\varepsilon^{4} \partial_{t}^{2} v_{\varepsilon}(x, t)\right)=\frac{\varepsilon^{2} a_{2}^{*}}{\left(a^{*}\right)^{2}}\left(a^{*} \partial_{x}^{6} v_{\varepsilon}(x, t)+\frac{a_{2}^{*}}{a^{*}} \varepsilon^{6} \partial_{t}^{2} \partial_{x}^{6} v_{\varepsilon}(x, t)\right)
\end{aligned}
$$

one obtains

$$
\begin{aligned}
& \left\|a_{2}^{*} \varepsilon^{2}\left(\frac{\varepsilon^{4}}{a^{*}} \partial_{t}^{2} \partial_{x}^{2} v_{\varepsilon}-\partial_{x}^{4} v_{\varepsilon}\right)(x, t)\right\|_{L^{2}\left(0, T ; L^{2}(\mathbb{R})\right)} \\
& \leq \frac{\varepsilon^{4}\left(a_{2}^{*}\right)^{2}}{\left(a^{*}\right)^{2}}\left(\left\|a^{*} \partial_{x}^{6} v_{\varepsilon}(x, t)\right\|_{L^{2}\left(0, T ; L^{2}(\mathbb{R})\right)}+\varepsilon^{6}\left\|\frac{a_{2}^{*}}{a^{*}} \partial_{t}^{2} \partial_{x}^{6} v_{\varepsilon}(x, t)\right\|_{L^{2}\left(0, T ; L^{2}(\mathbb{R})\right)}\right) \\
& \leq C \varepsilon^{4},
\end{aligned}
$$

where the last inequality is a consequence of Proposition 4.1. 
b),c) Expressions b) and c) have the same structure and can be handled in an analogous way. We consider expression b) and observe that

$$
\left(\frac{\varepsilon^{4}}{a^{*}} \partial_{t}^{2} \partial_{x}^{3} v_{\varepsilon}-\partial_{x}^{5} v_{\varepsilon}\right)(x, t)=\varepsilon^{6} \frac{a_{2}^{*}}{\left(a^{*}\right)^{2}} \partial_{t}^{2} \partial_{x}^{5} v_{\varepsilon}(x, t) .
$$

Consequently,

$$
\begin{aligned}
& \left\|a_{2}^{*} \varepsilon^{3} L_{1}\left(\frac{x}{\varepsilon}\right)\left(\frac{\varepsilon^{4}}{a^{*}} \partial_{t}^{2} \partial_{x}^{3} v_{\varepsilon}-\partial_{x}^{5} v_{\varepsilon}\right)(x, t)\right\|_{L^{2}\left(0, T ; L^{2}(\mathbb{R})\right)} \\
& \quad \leq a_{2}^{*} \varepsilon^{3}\left\|L_{1}\left(\frac{x}{\varepsilon}\right)\right\|_{L^{\infty}(\mathbb{R})}\left\|\left(\frac{\varepsilon^{4}}{a^{*}} \partial_{t}^{2} \partial_{x}^{3} v_{\varepsilon}-\partial_{x}^{5} v_{\varepsilon}\right)(x, t)\right\|_{L^{2}\left(0, T ; L^{2}(\mathbb{R})\right)} \\
& \quad \leq K \varepsilon^{9} \frac{\left(a_{2}^{*}\right)^{2}}{\left(a^{*}\right)^{2}}\left\|\partial_{t}^{2} \partial_{x}^{5} v_{\varepsilon}(x, t)\right\|_{L^{2}\left(0, T ; L^{2}(\mathbb{R})\right)} \\
& \quad \leq C \varepsilon^{4}
\end{aligned}
$$

due to Proposition 4.1.

d) The estimate of expression d) is a direct consequence of Proposition 4.1. One obtains

$$
\left\|\varepsilon^{4} a_{4}^{*} \partial_{x}^{6} v_{\varepsilon}(x, t)\right\|_{L^{2}\left(0, T ; L^{2}(\mathbb{R})\right)} \leq C \varepsilon^{4}
$$

e), f) Expressions e) and f) have the same structure. Taking into account $0<\alpha \leq$ $a\left(\frac{x}{\varepsilon}\right) \leq \beta$ and applying Proposition 4.1, one arrives at

$$
\left\|\varepsilon^{6} L_{6}\left(\frac{x}{\varepsilon}\right) a^{\varepsilon}(x) \partial_{x}^{8} v_{\varepsilon}(x, t)\right\|_{L^{2}\left(0, T ; L^{2}(\mathbb{R})\right)} \leq \varepsilon^{6} K \beta\left\|\partial_{x}^{8} v_{\varepsilon}(x, t)\right\|_{L^{2}\left(0, T ; L^{2}(\mathbb{R})\right)} \leq C \varepsilon^{6} .
$$

In the same manner,

$$
\left\|\varepsilon^{5} \partial_{x}^{7} v_{\varepsilon}(x, t)\left(a^{\varepsilon}(x)\left(L_{5}(.)+\partial_{y} L_{6}(.)\right)\left(\frac{x}{\varepsilon}\right)+\partial_{y}\left(a(.) L_{6}(.)\right)\left(\frac{x}{\varepsilon}\right)\right)\right\|_{L^{2}\left(0, T ; L^{2}(\mathbb{R})\right)} \leq C \varepsilon^{5}
$$

$\mathrm{g})$, h) The last two expressions can be estimated in an analogous way. Application of Proposition 4.1 leads to

$$
\begin{aligned}
& \left\|\varepsilon^{9}\left(\frac{a_{2}^{*}}{a^{*}} L_{3}\left(\frac{x}{\varepsilon}\right)+L_{5}\left(\frac{x}{\varepsilon}\right)\right) \partial_{t}^{2} \partial_{x}^{5} v_{\varepsilon}(x, t)\right\|_{L^{2}\left(0, T ; L^{2}(\mathbb{R})\right)} \\
\leq & C \varepsilon^{9} K\left\|\partial_{t}^{2} \partial_{x}^{5} v_{\varepsilon}(x, t)\right\|_{L^{2}\left(0, T ; L^{2}(\mathbb{R})\right)} \leq C \varepsilon^{4}, \\
& \left\|\varepsilon^{10}\left(\frac{a_{2}^{*}}{a^{*}} L_{4}\left(\frac{x}{\varepsilon}\right)+L_{6}\left(\frac{x}{\varepsilon}\right)\right) \partial_{t}^{2} \partial_{x}^{6} v_{\varepsilon}(x, t)\right\|_{L^{2}\left(0, T ; L^{2}(\mathbb{R})\right)} \leq C \varepsilon^{5} .
\end{aligned}
$$

Appendix B2: Estimates of the initial data. We have to show that

$$
\begin{gathered}
\left\|\partial_{x} u_{\varepsilon}(x, 0)-\partial_{x}\left(\mathcal{A}_{\varepsilon}\left(v_{\varepsilon}\right)\right)(x, 0)\right\|_{L^{2}(\mathbb{R})}^{2} \leq C \varepsilon^{4}, \\
\varepsilon^{4}\left\|\partial_{t} u_{\varepsilon}(x, 0)-\partial_{t}\left(\mathcal{A}_{\varepsilon}\left(v_{\varepsilon}\right)\right)(x, 0)\right\|_{L^{2}(\mathbb{R})}^{2} \leq C \varepsilon^{4} .
\end{gathered}
$$


Proof. Actually,

$$
\begin{aligned}
\left(\partial_{x}\left(\mathcal{A}_{\varepsilon}\left(v_{\varepsilon}\right)\right)-\partial_{x} u_{\varepsilon}\right)(x, 0) \\
=\varepsilon^{2} \partial_{y} L_{3}\left(\frac{x}{\varepsilon}\right) \partial_{x}^{3} c_{0}(x)+\varepsilon^{3}\left(L_{3}\left(\frac{x}{\varepsilon}\right)+\partial_{y} L_{4}\left(\frac{x}{\varepsilon}\right)\right) \partial_{x}^{4} c_{0}(x) \\
\quad+\varepsilon^{4}\left(L_{4}\left(\frac{x}{\varepsilon}\right)+\partial_{y} L_{5}\left(\frac{x}{\varepsilon}\right)\right) \partial_{x}^{5} c_{0}(x)+\varepsilon^{5}\left(L_{5}\left(\frac{x}{\varepsilon}\right)\right. \\
\left.\quad+\partial_{y} L_{6}\left(\frac{x}{\varepsilon}\right)\right) \partial_{x}^{6} c_{0}(x)+\varepsilon^{6} L_{6}\left(\frac{x}{\varepsilon}\right) \partial_{x}^{7} c_{0}(x)
\end{aligned}
$$

Since $c_{0}(x) \in C_{c}^{\infty}((-R, R))$, one directly obtains

$$
\left\|\varepsilon^{2} \partial_{y} L_{3}\left(\frac{x}{\varepsilon}\right) \partial_{x}^{3} c_{0}(x)\right\|_{L^{2}(\mathbb{R})}^{2} \leq \varepsilon^{4} K^{2}\left\|\partial_{x}^{3} c_{0}(x)\right\|_{L^{2}(\mathbb{R})}^{2} \leq C \varepsilon^{4}
$$

estimating the remaining terms in an analogous way. Similarly, we derive (5.11).

\section{Appendix C: Energy estimate for the long time wave equation.}

Lemma 5.5. Let $u \in L^{\infty}\left(0, T ; H^{2}(\mathbb{R})\right)$ with $\partial_{t} u \in L^{\infty}\left(0, T ; H^{1}(\mathbb{R})\right), \partial_{t}^{2} u \in L^{2}\left(0, T ; L^{2}(\mathbb{R})\right)$ be the unique solution to the long time wave equation

$$
\begin{aligned}
\varepsilon^{4} \partial_{t}^{2} u(x, t) & -\partial_{x}\left(a\left(\frac{x}{\varepsilon}\right) \partial_{x} u(x, t)\right)=f(x, t) \\
u(x, 0) & =a_{0}(x) \\
\partial_{t} u(x, 0) & =b_{0}(x)
\end{aligned}
$$

with $f \in L^{2}\left(0, T ; L^{2}(\mathbb{R})\right), a_{0} \in H^{2}(\mathbb{R})$ and $b_{0} \in H^{1}(\mathbb{R})$.

Then, there exists an $\varepsilon$-independent constant $C$ such that

$$
\begin{aligned}
& \left\|\partial_{x} u\right\|_{L^{\infty}\left(0, T ; L^{2}(\mathbb{R})\right)}^{2}+\varepsilon^{4}\left\|\partial_{t} u\right\|_{L^{\infty}\left(0, T ; L^{2}(\mathbb{R})\right)}^{2} \\
& \quad \leq C\left(\left\|\partial_{x} a_{0}\right\|_{L^{2}(\mathbb{R})}^{2}+\varepsilon^{4}\left\|b_{0}\right\|_{L^{2}(\mathbb{R})}^{2}+\frac{1}{\varepsilon^{4}}\|f\|_{L^{2}\left(0, T ; L^{2}(\mathbb{R})\right)}^{2}\right) .
\end{aligned}
$$

Proof. We multiply the time-scaled homogenization problem $(5.12)$ by $\partial_{t} u(x, t)$, integrate over $\mathbb{R}$ and apply integration by parts according to (3.12). Then

$$
\begin{aligned}
\varepsilon^{4} \frac{d}{d t}\left\|\partial_{t} u(\cdot, t)\right\|_{L^{2}(\mathbb{R})}^{2} & +\frac{d}{d t} \int_{\Omega} a\left(\frac{x}{\varepsilon}\right)\left(\partial_{x} u\right)^{2}(x, t) d x=2 \int_{\mathbb{R}} f(x, t) \partial_{t} u(x, t) d x \\
& \leq\left(\frac{4}{\varepsilon^{4}}\|f(\cdot, t)\|_{L^{2}(\mathbb{R})}^{2}+\varepsilon^{4}\left\|\partial_{t} u(\cdot, t)\right\|_{L^{2}(\mathbb{R})}^{2}\right)
\end{aligned}
$$

for almost every $t \in[0, T]$. Taking into account $0<\alpha \leq a\left(\frac{x}{\varepsilon}\right) \leq \beta$ and integrating 
over $(0, t)$, one arrives at

$$
\begin{aligned}
\varepsilon^{4}\left\|\partial_{t} u(\cdot, t)\right\|_{L^{2}(\mathbb{R})}^{2}+\alpha\left\|\partial_{x} u(\cdot, t)\right\|_{L^{2}(\mathbb{R})}^{2} \\
\leq \varepsilon^{4}\left\|b_{0}(x)\right\|_{L^{2}(\mathbb{R})}^{2}+\beta\left\|\partial_{x} a_{0}(x)\right\|_{L^{2}(\mathbb{R})}^{2}+\frac{4}{\varepsilon^{4}}\|f(x, t)\|_{L^{2}\left(0, T ; L^{2}(\mathbb{R})\right)}^{2} \\
\quad+\int_{0}^{t} \varepsilon^{4}\left\|\partial_{t} u(\cdot, \tau)\right\|_{L^{2}(\mathbb{R})}^{2} d \tau \\
\leq \varepsilon^{4}\left\|b_{0}(x)\right\|_{L^{2}(\mathbb{R})}^{2}+\beta\left\|\partial_{x} a_{0}(x)\right\|_{L^{2}(\mathbb{R})}^{2}+\frac{4}{\varepsilon^{4}}\|f(x, t)\|_{L^{2}\left(0, T ; L^{2}(\mathbb{R})\right)}^{2} \\
\quad+\int_{0}^{t} \varepsilon^{4}\left\|\partial_{t} u(\cdot, \tau)\right\|_{L^{2}(\mathbb{R})}^{2}+\alpha\left\|\partial_{x} u(\cdot, \tau)\right\|_{L^{2}(\mathbb{R})}^{2} d \tau
\end{aligned}
$$

Application of the Gronwall lemma finally leads to the claimed result.

\section{Acknowledgments}

The author is grateful to Ben Schweizer for suggesting the interesting topic, the constant support and numerous helpful discussions.

\section{References}

[1] G. Allaire. Homogenization and two-scale convergence. SIAM J. Math. Anal., 23(6):1482-1518, 1992.

[2] G. Allaire. Dispersive limits in the homogenization of the wave equation. Ann. Fac. Sci. Toulouse Math.(6), 12(4):415-431, 2003

[3] G. Allaire, M. Palombaro and J. Rauch. Diffractive behavior of the wave equation in periodic media: weak convergence analysis. Ann. Mat. Pura Appl.(4), 188(4):561-589, 2009

[4] S. Brahim-Otsmane, G. A. Francfort and F. Murat. Correctors for the homogenization of the wave and heat equations. J. Math. Pures Appl.(9), 71(3):197-231, 1992

[5] D. Cioranescu and P. Donato. An introduction to homogenization. Oxford Lecture series in Mathematics and its Applications, 1999.

[6] J. Fish, W. Chen and G. Nagai. Uniformly valid multiple spatial-temporal scale Modeling for wave propagation in heterogeneous Media. Mechanics of Advanced Materials and Structures, 8(2):81-99, 2001.

[7] J. Fish, W. Chen and G. Nagai. Non-local dispersive model for wave propagation in heterogeneous media: one-dimensional case. Internat. J. Numer. Methods Engrg., 54(3):331-346, 2002. 
[8] J. Fish and W. Chen. Space-time multiscale model for wave propagation in heterogeneous media. Comput. Methods Appl. Mech. Engrg., 193(45-47):4837-4856, 2004.

[9] J. Fish and W. Chen. A mathematical homogenization perspective of virial stress. Internat. J. Numer. Methods Engrg., 67(2):189-207, 2006.

[10] A.S. Fokas and B. Pelloni. Two-point boundary value problems for linear evolution equations. Math. Proc. Camb. Phil. Soc., 131(3):521-543, 2001.

[11] V. V. Jikov, S. M. Kozlov and A. O. Oleinik. Homogenization of differential operators and integral functionals. Springer-Verlag, Berlin, 1994 Translated from the Russian by G. A. Yosifian [G. A. Iosifyan]

[12] F. Santosa and W. Symes. A dispersive effective medium for wave propagation in periodic composites. SIAM J. Appl. Math., 51(4):984-1005, 1991.

[13] M. Mihailovici and B. Schweizer. Effective model for the cathode catalyst layer in fuel cells. Asymptot. Anal., 57(1-2):105-123, 2008.

[14] B. Schweizer. Averaging of flows with capillary hysteresis in stochastic porous media. European J. Appl. Math., 18(3):389-415, 2007.

[15] B. Schweizer. Homogenization of the Prager model in one-dimensional plasticity. Contin. Mech. Thermodyn., 20(8):459-477, 2009.

[16] B. Schweizer and M. Veneroni. Periodic homogenization of Prandtl-Reuss plasticity equations in arbitrary dimension. submitted, 2010. 


\section{Preprints ab 2008/14}

2010-05 Agnes Lamacz

Dispersive effective models for waves in heterogeneous media

2010-04 B. Schweizer and M. Veneroni

Periodic homogenization of Prandtl-Reuss plasticity equations in arbitrary dimension

2010-03 Holger Dette and Karl Friedrich Siburg and Pavel A. Stoimenov

A copula-based nonparametric measure of regression dependence

2010-02 René L. Schilling and Alexander Schnurr

The Symbol Associated with the Solution of a Stochastic Differential Equation

2010-01 Henryk Zähle

Rates of almost sure convergence of plug-in estimates for distortion risk measures

2009-16 Lorenz J. Schwachhöfer

Nonnegative curvature on disk bundles

2009-15 Iuliu Pop and Ben Schweizer

Regularization schemes for degenerate Richards equations and outflow conditions

2009-14 Guy Bouchitté and Ben Schweizer

Cloaking of small objects by anomalous localized resonance

2009-13 Tom Krantz, Lorenz J. Schwachhöfer

Extrinsically Immersed Symplectic Symmetric Spaces

2009-12 Alexander Kaplun

Continuous time Ehrenfest process in term structure modelling

2009-11 Henryk Zähle

Ein aktuarielles Modell für die Portabilität der Alterungsrückstellungen in der PKV

2009-10 Andreas Neuenkirch and Henryk Zähle

Asymptotic error distribution of the Euler method for SDEs with non-Lipschitz coefficients

2009-09 Karl Friedrich Siburg, Pavel A. Stoimenov

Regression dependence

2009-08 Wilfried Hazod

Continuous convolution hemigroups integrating a sub-multiplicative function

2009-07 Sergio Conti and Ben Schweizer

On optimal metrics preventing mass transfer

2009-06 Simon Castle, Norbert Peyerimhoff, Karl Friedrich Siburg

Billiards in ideal hyperbolic polygons

2009-05 Ludwig Danzer

Quasiperiodic Tilings - Substitution Versus Inflation

2009-04 Flavius Guiaş

Direct simulation of the infinitesimal dynamics of semi-discrete

approximations for convection-diffusion-reaction problems 
Existential definability of parallelism in terms of betweenness in Archimedean ordered affine geometry in one space dimension

\section{Henryk Zähle}

Approximation of SDEs by population-size-dependent

Galton-Watson processes

2008-25 Winfried Hazod

Mehler semigroups, Ornstein-Uhlenbeck processes and background driving Lévy processes on locally compact groups and on hypergroups

2008-24 Karl Friedrich Siburg, Pavel A. Stoimenov

Symmetry of functions and exchangeability of random variables

2008-23 Ina Kirsten Voigt

Voronoi Cells of Discrete Point Sets

$2008-22$

$2008-21$

Michael Lenzinger and Ben Schweizer

Effective reaction rates of a thin catalyst layer

\section{Michael Voit}

Bessel convolutions on matrix cones: Algebraic properties and random walks

\section{Margit Rösler and Michael Voit}

Limit theorems for radial random walks on $p \times q$-matrices as

$p$ tends to infinity

2008-19 Michael Voit

Central Limit Theorems for Radial Random Walks on

$p \times q$ Matrices for $p \rightarrow \infty$

2008-18 Michael Voit

Limit theorems for radial random walks on homogeneous spaces with growing dimensions

2008-17 Ansgar Steland and Henryk Zähle

Sampling inspection by variables: nonparametric setting

2008-16 Guy Bouchitté and Ben Schweizer

Homogenization of Maxwell's equations with split rings

2008-15 Wilfried Hazod

Multiple selfdecomposable laws on vector spaces and on groups:

The existence of background driving processes

2008-14 Wilfried Hazod

Mixing of generating functionals and applications to (semi-)stability of probabilities on groups 